\title{
Antibiotic effects on gut microbiota and metabolism are host dependent
}

\author{
Shiho Fujisaka, ${ }^{1}$ Siegfried Ussar, ${ }^{1,2}$ Clary Clish, ${ }^{3}$ Suzanne Devkota, ${ }^{4}$ Jonathan M. Dreyfuss, ${ }^{5,6}$ Masaji Sakaguchi, ${ }^{1}$ Marion Soto, ${ }^{1}$ \\ Masahiro Konishi, ${ }^{1}$ Samir Softic, ${ }^{1,7}$ Emrah Altindis, ${ }^{1}$ Ning Li, ${ }^{8}$ Georg Gerber, ${ }^{8}$ Lynn Bry, ${ }^{8}$ and C. Ronald Kahn ${ }^{1}$ \\ 'Section of Integrative Physiology and Metabolism, Joslin Diabetes Center and Harvard Medical School, Boston, Massachusetts, USA. 2nstitute for Diabetes and Obesity, Helmholtz Zentrum München, \\ Neuherberg, Germany; German Center for Diabetes Research (DZD), Munich, Germany. ${ }^{3}$ Broad Institute of MIT and Harvard, Cambridge, Massachusetts, USA. ${ }^{4}$ Section of Pathophysiology and Molecular \\ Pharmacology, Joslin Diabetes Center and Harvard Medical School, Boston, Massachusetts, USA. ${ }^{5}$ Bioinformatics Core, Joslin Diabetes Center and Harvard Medical School, Boston, Massachusetts, USA. \\ ${ }^{6}$ Department of Biomedical Engineering, Boston University, Boston, Massachusetts, USA. ${ }^{7}$ Boston Children's Hospital, Boston, Massachusetts, USA. ${ }^{8}$ Center for Clinical and Translational Metagenomics, \\ Department of Pathology, Brigham and Women's Hospital, Boston, Massachusetts, USA.
}

\begin{abstract}
Interactions of diet, gut microbiota, and host genetics play important roles in the development of obesity and insulin resistance. Here, we have investigated the molecular links between gut microbiota, insulin resistance, and glucose metabolism in 3 inbred mouse strains with differing susceptibilities to metabolic syndrome using diet and antibiotic treatment. Antibiotic treatment altered intestinal microbiota, decreased tissue inflammation, improved insulin signaling in basal and stimulated states, and improved glucose metabolism in obesity- and diabetes-prone C57BL/6] mice on a highfat diet (HFD). Many of these changes were reproduced by the transfer of gut microbiota from antibiotic-treated donors to germ-free or germ-depleted mice. These physiological changes closely correlated with changes in serum bile acids and levels of the antiinflammatory bile acid receptor Takeda G protein-coupled receptor 5 (TCR5) and were partially recapitulated by treatment with a TCR5 agonist. In contrast, antibiotic treatment of HFD-fed, obesity-resistant 129S1 and obesity-prone 12956 mice did not improve metabolism, despite changes in microbiota and bile acids. These mice also failed to show a reduction in inflammatory gene expression in response to the TCR5 agonist. Thus, changes in bile acid and inflammatory signaling, insulin resistance, and glucose metabolism driven by an HFD can be modified by antibiotic-induced changes in gut microbiota; however, these effects depend on important interactions with the host's genetic background and inflammatory potential.
\end{abstract}

\section{Introduction}

Gut microbiota affect the host's energy balance, glucose and lipid metabolism, and the immune response (1). Obesity, type 2 diabetes, and metabolic syndrome are all associated with changes in intestinal microbial communities and reduced bacterial diversity (2). Causal effects of the microbiota have been suggested by experiments showing that colonization of germ-free (GF) mice with gut microbiota from obese mice (3) or humans (4) can result in increased fat mass in the recipients. Likewise, changes in gut microbiota induced by antibiotics $(5,6)$, cohousing animals $(7)$, or changing the breeding/ rearing environment (8) can affect the response to high-fat dietinduced (HFD-induced) obesity and insulin resistance. The effect of the microbiome on metabolism is also modified by the genetics of the host (8) and by environmental factors such as dietary intake (9). Exactly how gut microbiota interact with host genetics and affect the metabolic phenotype is still incompletely defined. Multiple mechanisms have been suggested including the effects of bacterially released LPSs (10), the metabolism of short-chain fatty acids and bile acids $(11,12)$, and the effects on the host's immune system (13).

Among strains of mice and individual humans, a wide range of responses is observed in the development of obesity and insulin

Conflict of interest: The authors have declared that no conflict of interest exists. Submitted: January 22, 2016; Accepted: September 15, 2016.

Reference information: J Clin Invest. 2016;126(12):4430-4443. doi:10.1172/JCI86674. resistance with exposure to high-calorie or high-fat diets $(14,15)$. For example, C57BL/6J (B6J) mice from The Jackson Laboratory and 129S6/SvEvTac (129T) mice from Taconic Farms are obesity prone, whereas a genetically related 129 substrain from The Jackson Laboratory (129S1/SvImJ, abbreviated herein as 129J) is obesity resistant. The difference between the 129 substrains from these 2 vendors is due, at least in part, to differences in gut microbiota, as housing these mice in a common environment ameliorates their propensity to develop obesity and glucose intolerance (8). Adipose tissue and systemic inflammation are observed in many disorders associated with insulin resistance including obesity, type 2 diabetes, and metabolic syndrome $(16,17)$, and targeting inflammation has been explored as a treatment for metabolic disorders (18). While there is evidence for some interplay between the gut microbiota and obesity-induced inflammation $(8,19,20)$, the molecular links between these processes and the role of host genetics have not been fully elucidated.

The aim of this study was to dissect the molecular links between gut microbiota, insulin resistance, and glucose metabolism using inbred mouse strains that differ in their propensity to develop metabolic syndrome. To this end, we challenged B6J, 129T, and 129J mice with an HFD, while simultaneously treating some animals with vancomycin, a nonabsorbed antibiotic that targets gram-positive bacteria, or metronidazole, an absorbed antibiotic that targets anaerobic bacteria. Though all 3 strains showed 


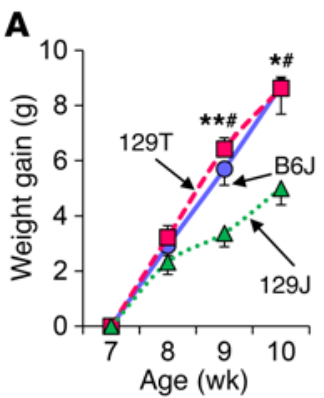

E

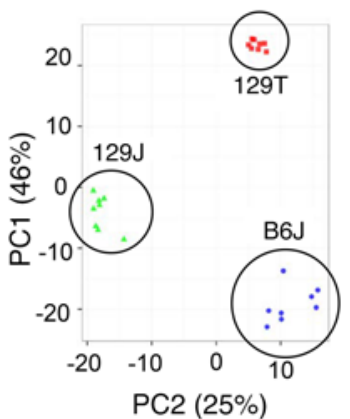

I

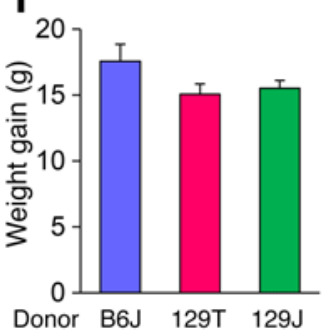

Recipient GF B6J mice
B

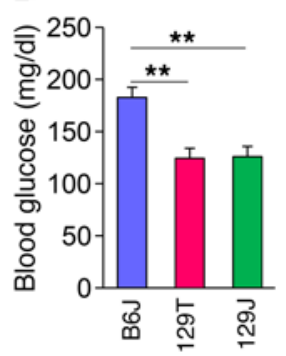

F

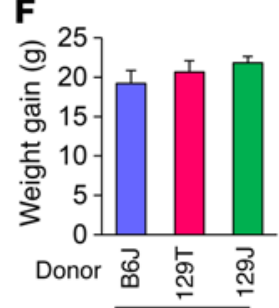

Recipient $\overline{\mathrm{B} 6 \mathrm{~J} \text { after } \mathrm{Ab}}$

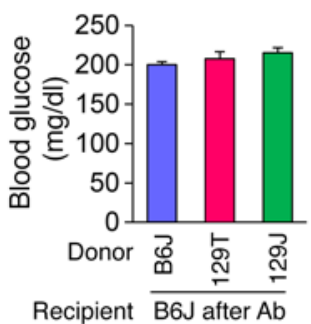

C

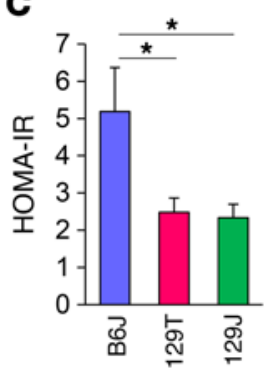

G
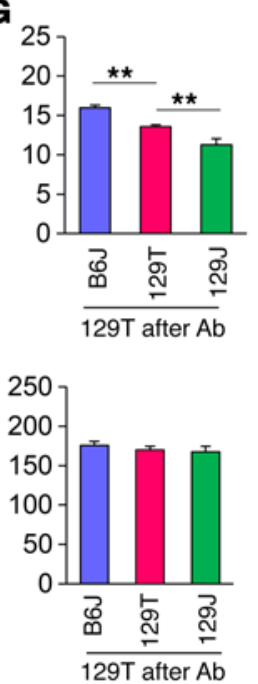

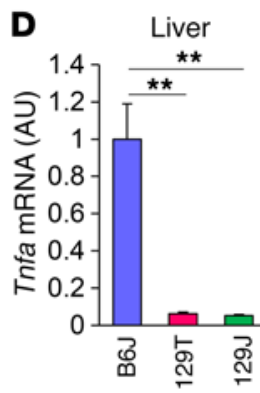

H
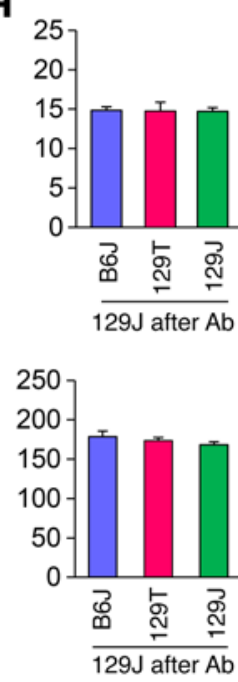

J

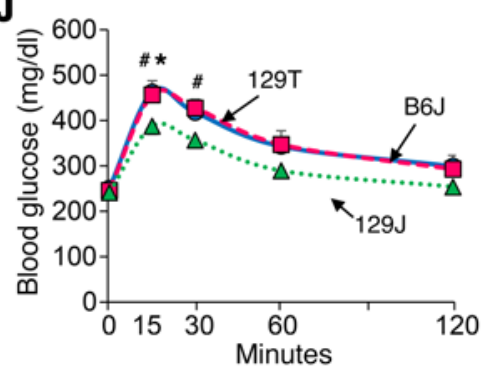

Figure 1. Microbiota affect adiposity and glucose metabolism depending on the host genetics. (A) Weight gain of B6J (circles), 129T (squares), and 129] (triangles) mice on an HFD. ${ }^{\#} P<0.05$ (129T vs. 129)); ${ }^{*} P<0.05$ and ${ }^{* *} P<0.01$ (B6] vs. 129)), by ANOVA, followed by Tukey-Kramer post-hoc. (B) Blood glucose levels in the random fed state at 11 weeks of age ( $n=4$ per group). (C) Calculated HOMA-IR for B6J, 129T, and 129] mice after 8 weeks on an HFD ( $n=8$ per group). (D) Tnfa mRNA in liver from mice fed an HFD for 9 weeks as determined by qPCR ( $n=8$ per group). (E) PCA of fecal 165 rRNA sequencing data for HFD-fed mice of the 3 strains at 16 weeks of age $(n=8$ per group). $\mathrm{PC}$, principle coordinate 1; PC2, principle coordinate 2 . (F-H)

Graphs show weight gain of HFD-fed B6J (F), 129T (G), and 129J (H) mice 10 weeks after undergoing transfer of cecal bacteria from B6), 129T, and 129] mice ( $n=6$ per group). Graphs show blood glucose levels (fed) of recipient B6J (F), 129T (C), and 129J (H) mice measured 1 week after bacterial transfer. ${ }^{*} P<0.05$ and ${ }^{* *} P<0.01$, by ANOVA, followed by Tukey-Kramer post-hoc. (I) Weight gain and (J) OCTT for HFD-fed, GF B6) mice colonized with cecal bacteria from B6) (circle), 129T (square), and 129J (triangle) mice measured 2 weeks after transfer $(n=7-10) .{ }^{\#} P<0.05$ (129T vs. 129 )); ${ }^{*} P<0.05$ (B6) vs. 129)), by ANOVA, followed by Tukey-Kramer post-hoc. dramatic changes in their gut microbial communities following antibiotic treatment, only the obesity-prone $\mathrm{B} 6 \mathrm{~J}$ mice had a reduction in inflammatory markers as well as improved insulin signaling and glucose metabolism when given antibiotics. These improvements were associated with changes in serum bile acids, especially deoxycholic acid (DCA) and taurodeoxycholic acid (TDCA), and restoration of levels of the bile acid receptor Takeda G proteincoupled receptor 5 (TGR5) in the liver, which was decreased by an HFD. Likewise, treatment with a TGR5 agonist reduced inflammatory gene expression and response to LPS only in B6J mice. Thus, the effects of antibiotics on improved insulin signaling and the metabolic state depend on an interaction of the gut microbiome, bile acid metabolism, and the systemic inflammatory response, all of which are dependent on host genetics.

\section{Results}

Effects of gut microbiota on adiposity and glucose metabolism in different inbred mouse strains. To assess the effect of host genetics on the metabolic response to HFD-induced obesity, 7-week-old male inbred B6J, 129T, and 129J mice were challenged with an HFD (60\% of calories from fat). As previously described (8), while all 3 strains of mice had similar food intake, by 2 weeks into the study, B6J and $129 \mathrm{~T}$ mice had gained significantly more weight than did 129J mice on the same diet (Figure 1A). The response to weight gain, however, was different between B6J and 129T mice. B6J mice developed hyperglycemia, hyperinsulinemia, and marked insulin resistance as measured by homeostatic model assessment of insulin resistance (HOMA-IR) (Figures 1, B and C), whereas, despite comparable weight gains, $129 \mathrm{~T}$ mice had lower blood glucose and HOMA-IR levels that were similar to those of 129J mice, which remained relatively lean and insulin sensitive (Figure 1, B and C). This higher level of insulin sensitivity of 129T and 129J mice on an HFD correlated with lower expression levels of Tnfa in the liver compared with levels detected in B6J mice (Figure 1D).

We have previously shown that the different metabolic responses to an HFD in these 3 strains of mice are in part due to 

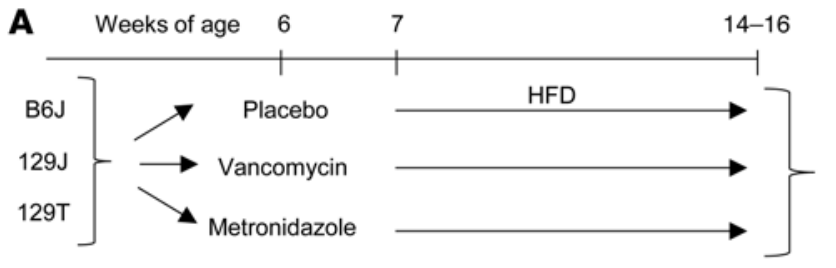

Physiological characterization

Microbiome analysis by $16 \mathrm{~S}$ rRNA sequencing
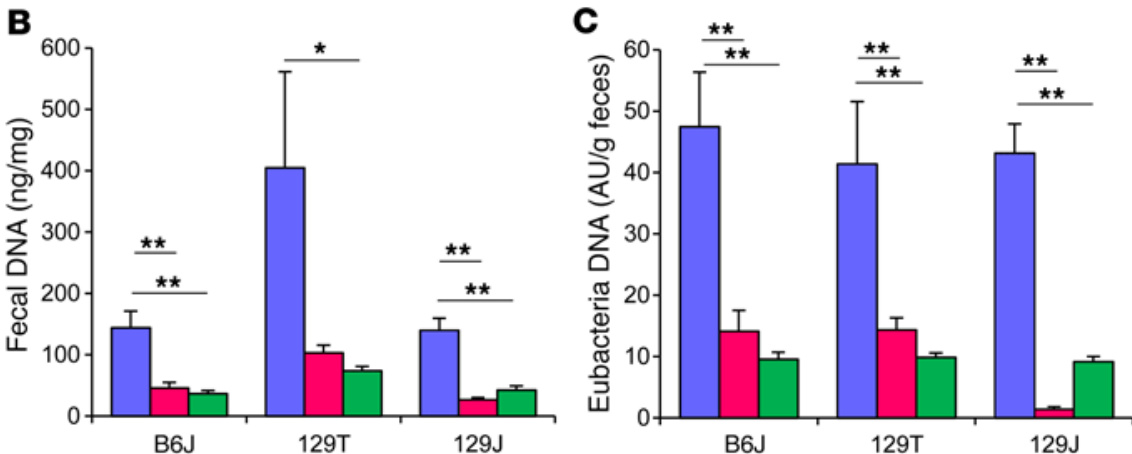

E
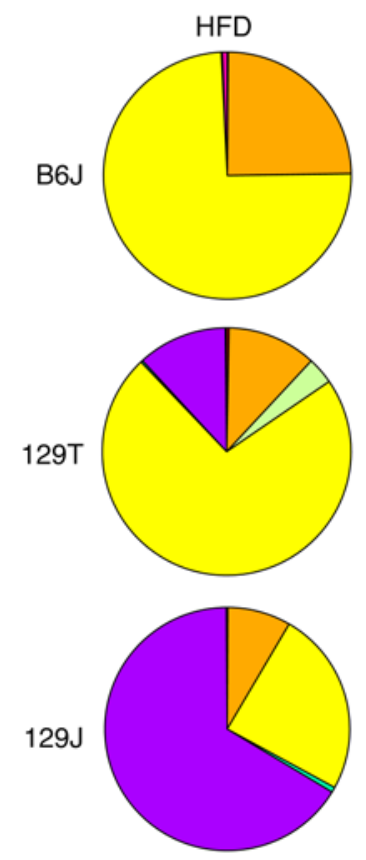
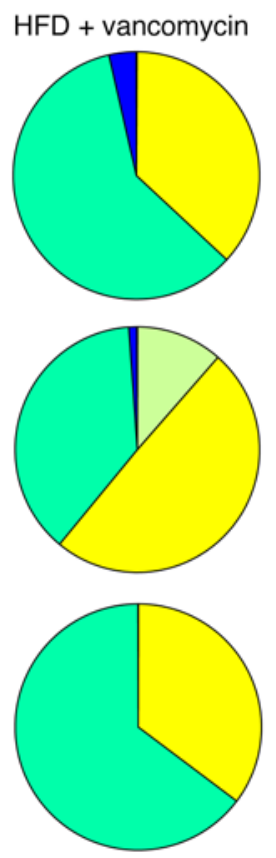
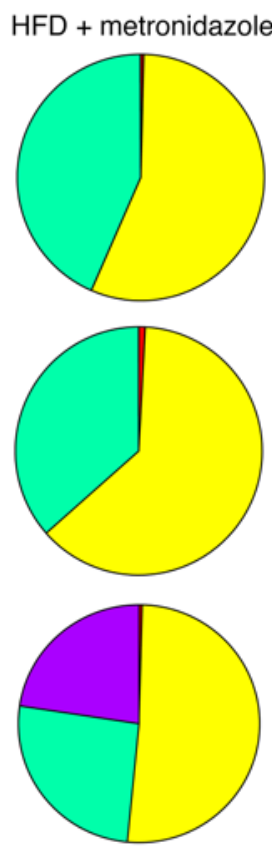

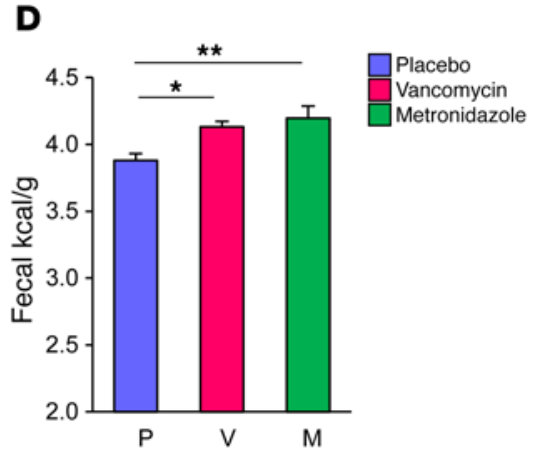

D
Actinobacteria

Bacteroidetes

$\square$ Deferribacteres

$\square$ Firmicutes

$\square$ Proteobacteria

Spirochaetes

$\square$ Tenericutes

$\square$ Verrucomicrobia

Unclassified

Figure 2. Antibiotic treatment decreases biomass of the gut bacteria and dramatically modulates microbiota composition. (A) Schematic overview of the study design for antibiotic modification of the gut microbiota using 3 strains of mice. (B) DNA levels isolated from fecal samples and (C) eubacteria DNA levels measured by qPCR normalized by fecal weight after 1 week of antibiotic treatment ( $n=8$ per group). (D) Energy content measured by bomb calorimetry of fecal samples from B6J mice after 5 weeks of antibiotic treatment $(n=8)$. P, placebo; V, vancomycin; M, metronidazole. (E) Representation of bacterial phyla in the fecal microbiota of mice from each group $(n=8)$ at 14 weeks of age after 8 weeks of antibiotic treatment. ${ }^{*} P<0.05$ and ${ }^{* *} P<0.01$ by ANOVA, followed by Tukey-Kramer post-hoc.

differences in their gut microbiota (8). Indeed, principal component analysis (PCA) of gut microbiota, as assessed by $16 \mathrm{~S}$ rRNA sequencing of the $\mathrm{V} 4$ region, showed clear differences in the microbial communities of these mice (Figure 1E). To determine whether the metabolic differences among the strains were related to these differences, we transferred cecal bacteria from mice of each of the 3 different strains into B6J (Figure 1F), 129T (Figure 1G), and 129J (Figure 1H) mice, whose endogenous gut microbes were markedly reduced by a 3-day treatment with an antibiotic cocktail consisting of ampicillin, vancomycin, neomycin, and metronidazole and chal- lenged them with an HFD for 10 weeks. On the 129T genetic background, mice receiving microbiota from B6J donors gained even more weight than did mice receiving microbiota from $129 \mathrm{~T}$ donors, whereas mice that received bacteria from 129J mice gained significantly less weight than did those receiving bacteria from $129 \mathrm{~T}$ mice (Figure 1G). This difference in weight gain was not observed when the recipient mice were either of the B6J strain (Figure 1F) or the 129J strain (Figure 1H), further indicating the importance of the host in the response to the microbiome. It is also worth noting that B6J mice gained more weight and showed higher glucose levels, 
A

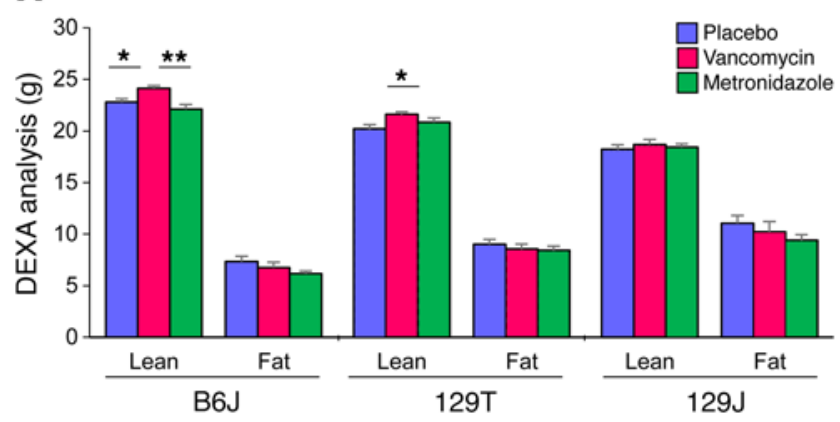

B

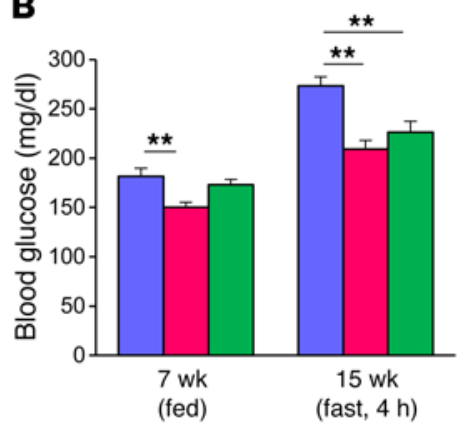

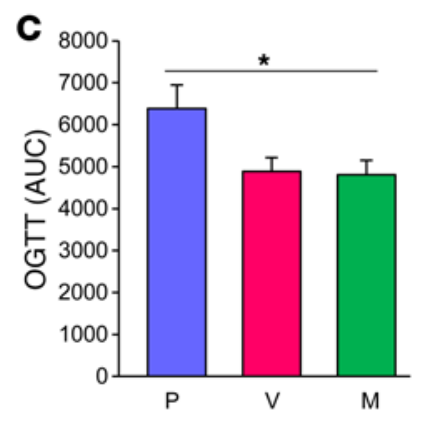

D

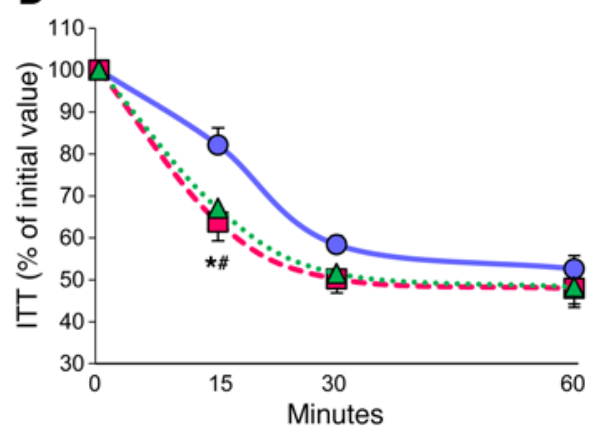

E

Insulin $\mathrm{p}-\mathrm{IR}$ p-AKT

AKT p-ERK

ERK GAPDH

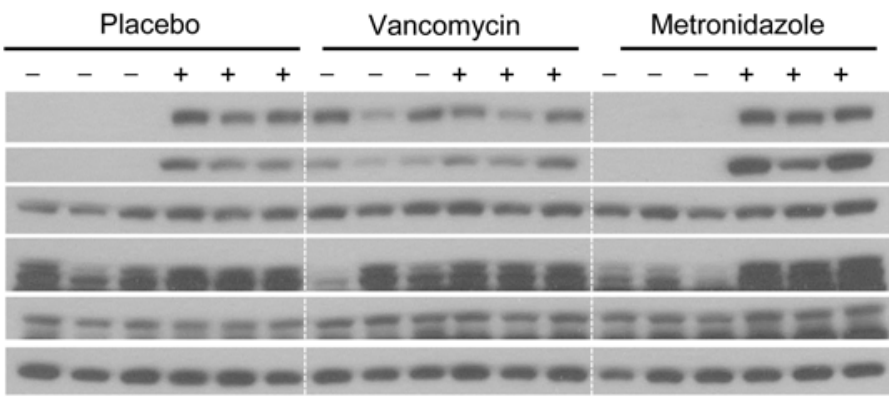

$\mathbf{F}$

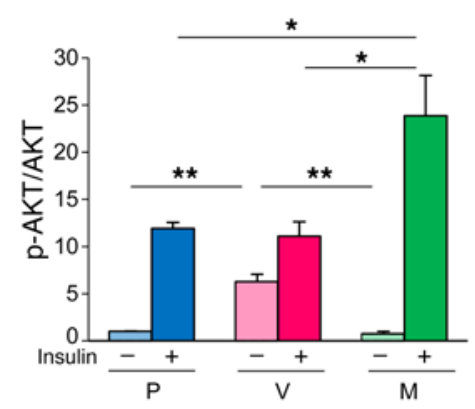

G

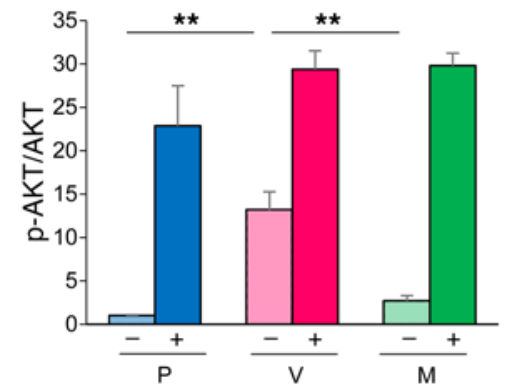

H

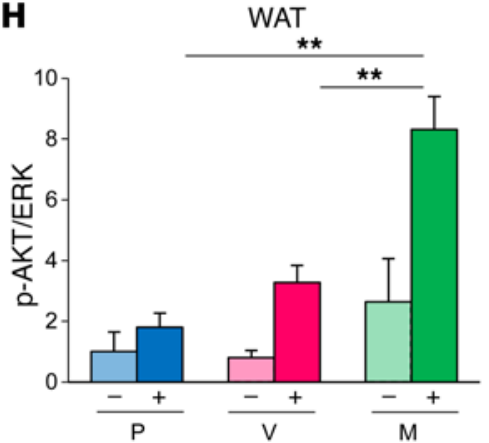

Figure 3. Antibiotic modification of the gut microbiota improves glucose metabolism with improved insulin signaling in HFD-fed B6) mice. (A) Total fat and lean mass were assessed by DEXA for HFD-fed B6J, 129T, and 129J mice treated with placebo, vancomycin, or metronidazole for 7 weeks ( $n=16)$. (B) Blood glucose levels of HFD-fed B6) mice in the fed (7 weeks old) or 4-hour-fasted state (15 weeks old) $(n=8)$. (C) AUC of blood glucose levels during an OCTT of 19-week-old HFD-fed B6) mice treated with placebo, vancomycin, or metronidazole (12 weeks on the HFD diet; 13 weeks on antibiotics) ( $n=6$ ). (D) ITT of 12-week-old HFD-fed B6J mice treated with placebo, vancomycin, or metronidazole (7 weeks on the HFD; 8 weeks on antibiotics) ( $n=16$ ). (E) Western blots for insulin signaling in liver extracts from 16-week-old B6) mice treated with $5 \mathrm{U}$ insulin via the vena cava ( 9 weeks on the HFD; 10 weeks on antibiotics) $n=3$. (F-H) Quantitation of AKT phosphorylation (p-AKT) normalized by total AKT in liver (F), muscle (G), and adipose tissue (H) extracts from B6J mice treated with placebo, vancomycin, or metronidazole) $(n=3) .{ }^{*} P<0.05$ and ${ }^{* *} P<0.01$, by ANOVA, followed by Tukey-Kramer post-hoc.

regardless of the bacterial donors, consistent with this being the most obesity- and diabetes-prone mouse of these 3 strains. Random fed blood glucose levels in mice from each recipient group were not different in any of the strains, suggesting that the failure of $129 \mathrm{~T}$ mice to develop hyperglycemia despite high weight gain in response to an HFD is due to the effects of the genetic background, whereas differences in the tendency toward obesity are more closely linked to differences in the gut microbiota.

To further explore the role of the host's genetic background in the response to changing gut microbiota, we also performed cecal bacterial transfer from mice of each strain into GF B6J mice and challenged the latter with an HFD. Five-week-old male GF mice were started on an irradiated HFD. After 1 week, transfer of the cecal contents from the 3 strains of donor mice was performed. The colonized mice were continuously fed the same HFD for 8 more weeks. Mice on the B6J background that received bacteria from HFD-fed B6J mice again gained more weight than did mice that received bacteria from either 129J or 129T mice, however, this did not quite reach statistical significance $(P=0.15)$ (Figure 1I). More important, the $\mathrm{B} 6 \mathrm{~J}$ recipients colonized with bacteria from both B6J and $129 \mathrm{~T}$ mice had worse glucose tolerance than did those colonized with bacteria from $129 \mathrm{~J}$ mice (Figure 1J). These results suggest that $129 \mathrm{~T}$ mice are prone to obesity, in part, because of their gut microbiota but remain glucose tolerant because they are genetically insulin sensitive. However, if their microbiome was transferred into diabetes-prone, HFD-fed B6J mice, the recipi- 
ent mice showed signs of glucose intolerance, again indicating an interaction of gut bacteria with host genetics that affects adiposity and glucose metabolism.

Antibiotic treatment dramatically modulates gut microbiota composition. To dissect the relationship between gut microbiota and the host's metabolism, we challenged B6J, 129T, and 129J mice with an HFD, while modifying the microbiome by treatment with either vancomycin or metronidazole (Figure 2A). Vancomycin is a nonabsorbable antibiotic that kills primarily gram-positive organisms, whereas metronidazole targets anaerobic organisms and is absorbable. These are the 2 antibiotics also used most frequently in patients with Clostridium difficile infection. Quantitative PCR (qPCR) analysis showed that antibiotic treatment reduced total and eubacteria DNA levels in the feces by $70 \%$ to $90 \%$ in all 3 strains of mice (Figure 2, B and C), and this was associated with an increase in the total amount of energy in the feces as measured by bomb calorimetry $(P=0.005)$ (Figure 2D).

16S rRNA sequencing analysis of fecal samples was performed to determine the bacterial composition of each mouse strain after 7 weeks on an HFD, with or without antibiotic treatment. Despite their different genetic backgrounds and breeding sites, the gut microbiota of both B6J and 129T mice on an HFD were dominated by Firmicutes (74\% in B6J and $72 \%$ in $129 \mathrm{~T}$ mice), whereas in 129J mice, Verrucomicrobia accounted for $66 \%$ of the bacterial sequences. Vancomycin treatment reduced the relative abundance of Firmicutes in B6J mice to $37 \%(P=0.009)$ and in $129 \mathrm{~T}$ mice to $50 \%$ of the untreated HFD levels $(P=0.003)$, and in both cases, this was associated with an increase in the relative abundance of Proteobacteria (Figure 2E). Interestingly, despite the difference in initial bacterial composition in 129J mice, metronidazole and vancomycin markedly reduced Verrucomicrobia from $66 \%$ to $0 \%(P=0.002)$ and $23 \%(P=0.007)$, respectively, leaving only Proteobacteria and Firmicutes (Figure 2E and Supplemental Figure 1A; supplemental material available online with this article; doi:10.1172/JCI86674DS1). PCA showed that, despite initial differences in microbiota among these 3 strains, the major determinant of the microbial landscape was antibiotic treatment (Supplemental Figure 1B). Nonetheless, even on the same HFD and the same antibiotic, each strain had unique microbiotial communities.

Modification of gut microbiota by antibiotics improves glucose metabolism and insulin signaling in B6J mice. In the obesity-prone B6J mice on an HFD, the vancomycin-treated mice gained significantly more weight than did the metronidazole-treated animals, while placebo-treated mice had weight gains intermediate to those of the 2 groups (Supplemental Figures 2A). Vancomycin-treated B6J mice exhibited a significant increase in lean mass $(22.8 \pm 0.33 \mathrm{~g}$ to $24.1 \pm 0.27 \mathrm{~g} ; P=0.002)$, with no change in fat mass as assessed by dual-energy $\mathrm{x}$-ray absorptiometry (DEXA) (Figure 3A). By comparison, there were no significant differences in weight gain in either the $129 \mathrm{~T}$ or $129 \mathrm{~J}$ mice during antibiotic treatment, although there was a small increase in lean mass in the 129T mice on vancomycin (Supplemental Figure 2, B and C, and Figure $3 \mathrm{~A}$ ). Food intake was not altered by antibiotic treatment in mice of any of the strains (Supplemental Figures 2, D and F).

Both vancomycin and metronidazole lowered blood glucose levels (Figure 3B) and improved glucose tolerance during an oral glucose tolerance test (OGTT) (Figure $3 \mathrm{C}$ ) in B6J mice on an HFD and resulted in improved insulin sensitivity as assessed by an i.p. insulin tolerance test (ITT) (Figure 3D). This was due to improved insulin signaling in target tissues of the antibiotic-treated mice (Figure 3, E-H, and Supplemental Figure 3, A and B), with no change in serum insulin levels (Supplemental Figure 3, G and H). Indeed, in both liver and muscle, vancomycin treatment enhanced basal phosphorylation of the insulin receptor (IR), AKT (Ser473), and ERK, even in animals that received no exogenous insulin. Metronidazole also increased AKT phosphorylation, but only in response to insulin (Figure 3, E-G, and Supplemental Figure 3B). Enhanced insulin-stimulated phosphorylation of IR and AKT in vancomycinand metronidazole-treated mice was also observed in epididymal adipose tissue, but in this tissue, there were no changes in basal phosphorylation (Figure 3H and Supplemental Figure 3A). By contrast, in $129 \mathrm{~T}$ and $129 \mathrm{~J}$ mice, antibiotic treatment did not change glucose levels, insulin levels, or insulin sensitivity (Supplemental Figure 3, C-F, and Supplemental Figure 3, G and H). Thus, metronidazole and vancomycin treatment improved glucose metabolism and insulin signaling only in HFD-fed B6J mice.

Transplantation of gut bacteria transfers improved insulin signaling and glucose metabolism. To determine whether the improved metabolic phenotype observed in antibiotic-treated B6J mice was secondary to changes in gut microbiota, we performed cecal microbiota transfer from vancomycin- or metronidazole-treated donor mice into microbiota-depleted B6J recipient mice as described above (Supplemental Figure 4A). During the subsequent 12 days, the BW of recipient mice was stable and did not differ between control mice and those that received microbiota from antibiotic-treated animals (Supplemental Figure 4B). However, the recipient mice that received metronidazole-treated cecal content had significantly improved glucose levels 7 days after transfer, and this trend continued for the remainder of the experiment (Figure 4A). The mice that received microbiota from vancomycin-treated mice also had lower glucose levels, but these did not reach statistical significance. OGTT also showed improved glucose tolerance in the groups that received microbiota from the antibiotic-treated groups compared with pre-transfer levels in the same animals (Figure 4, B-D). A similar effect was observed during an i.p. glucose tolerance test (GTT), indicating that this was a systemic effect rather than an effect localized to the gastrointestinal tract (Supplemental Figure 4, C-E). More important, like mice directly treated with antibiotics, mice that received the microbiota from antibiotic-treated animals also showed improved insulin-stimulated IR and AKT phosphorylation in liver and muscle (Figure 4, E and F).

To further investigate these phenomena, we performed a second bacterial transfer experiment in HFD-fed, GF B6J mice (Supplemental Figure 4F). Again, we observed significant improvement in AKT phosphorylation (p-AKT) in the livers of mice that received gut bacteria from vancomycin-treated mice (Figure $4 G$ ). OGTT showed improved glucose tolerance in recipient mice with vancomycin- or metronidazole-treated donors (Figure $4 \mathrm{H}$ ). Thus, antibiotic modification of gut microbiota improved glucose metabolism and insulin signaling in HFD-fed B6J mice, but not in $129 \mathrm{~T}$ or $129 \mathrm{~J}$ mice, and these effects were in large part transferable to both GF mice and mice whose endogenous microbiota were markedly reduced by prior antibiotic treatment. 
A
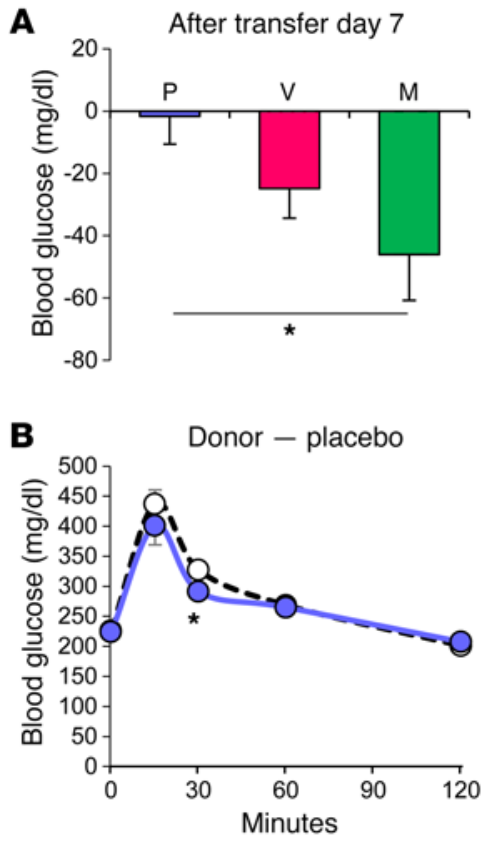

E

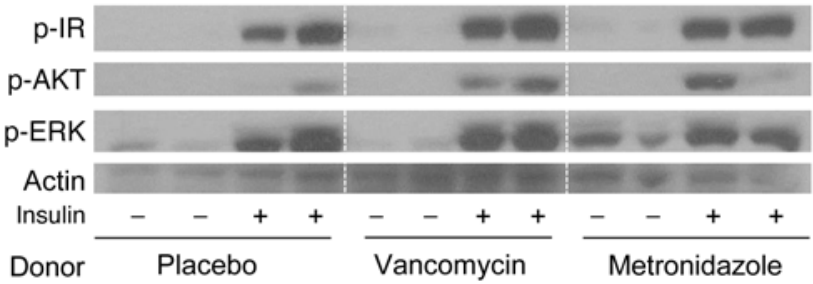

G
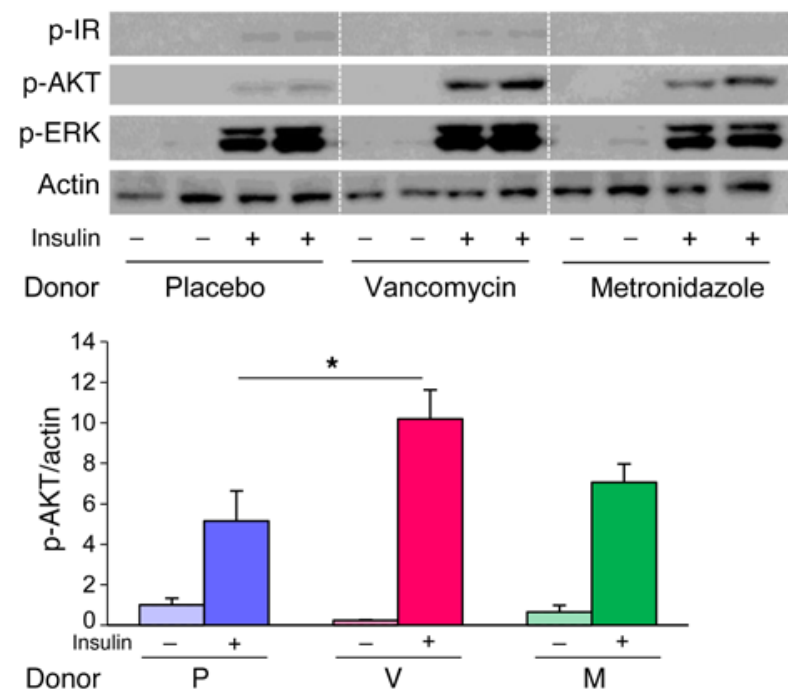

After transfer day 9
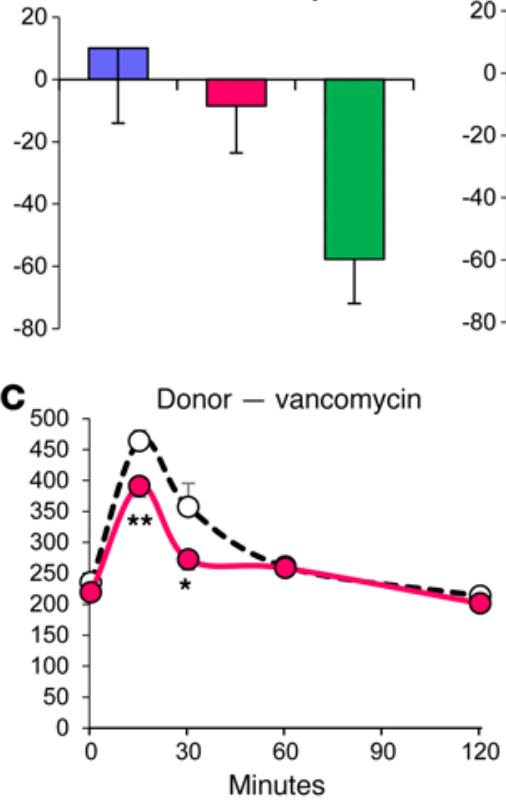

$\mathbf{F}$
After transfer day 11
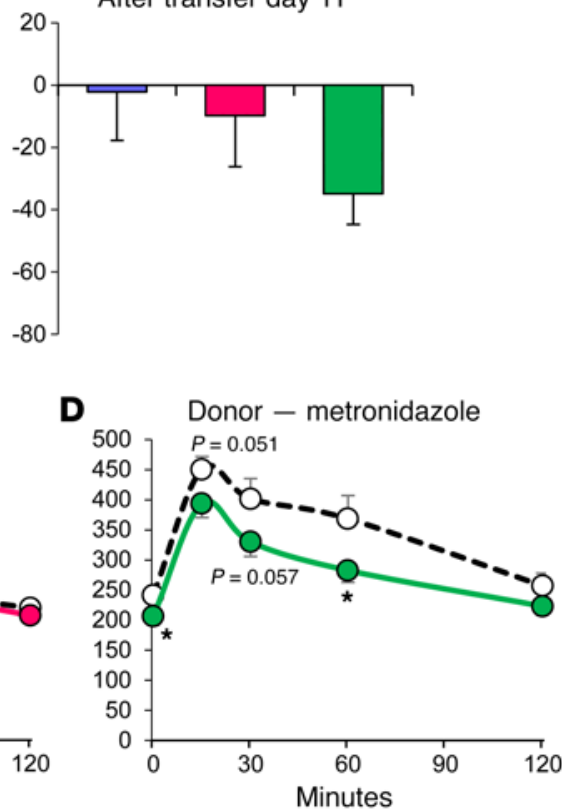

Muscle

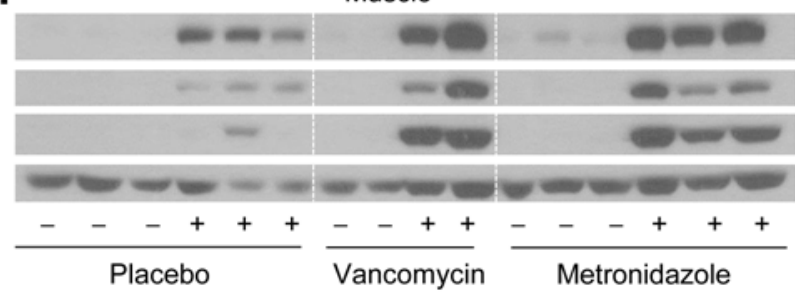

H

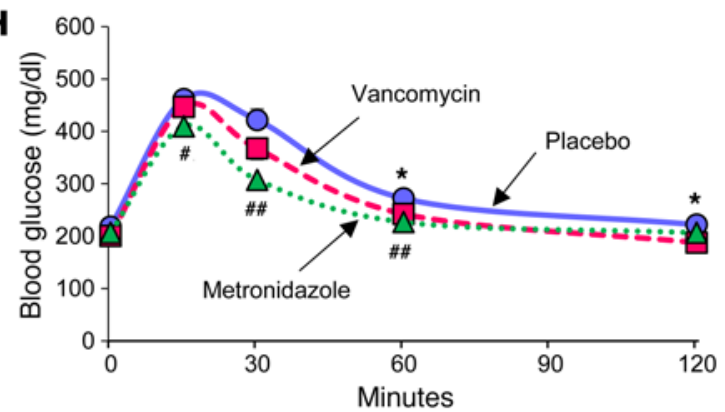

Figure 4. Improvement of glucose metabolism by antibiotic-modified bacteria is transferable. (A) Differences between 4-hour fasting blood glucose levels measured at $1 \mathrm{pm}$ in an HFD-fed B6) mice before and after bacterial transfer (days 7, 9, and 11) from donor HFD-fed B6) mice treated with placebo, vancomycin, or metronidazole for 1 week ( $n=6$ per group). (B-D) OCTT of the HF-fed B6) recipient mice performed before (white circles) and after (solid circles) bacterial transfer (day 11) from mice treated with placebo (B), vancomycin (C), or metronidazole (D) $(n=6) .{ }^{*} P<0.05$ and ${ }^{* *} P<0.01$, by unpaired 2-tailed t test. (E and $\mathbf{F}$ ) Western blots for insulin signaling in liver (E) and muscle (F) of the recipient mice. (G) Western blots for insulin signaling in the liver of HFD-fed, GF B6] mice colonized with cecal bacteria from HFD-fed B6] mice treated with placebo, vancomycin, or metronidazole, measured 2 weeks after transfer. Graph shows quantitation of p-AKT protein normalized by actin $(n=4-6)$. (H) OCTT of HFD-fed, GF B6) mice colonized with cecal bacteria from HFD-fed B6] mice treated with placebo (circles), vancomycin (squares), or metronidazole (triangles) $(n=7-9)$. ${ }^{*} P<0.05$, for placebo versus vancomycin; ${ }^{\#} P<0.05$ and ${ }^{\# \#} P<0.01$, for placebo versus metronidazole, by ANOVA, followed by Tukey-Kramer post-hoc. 

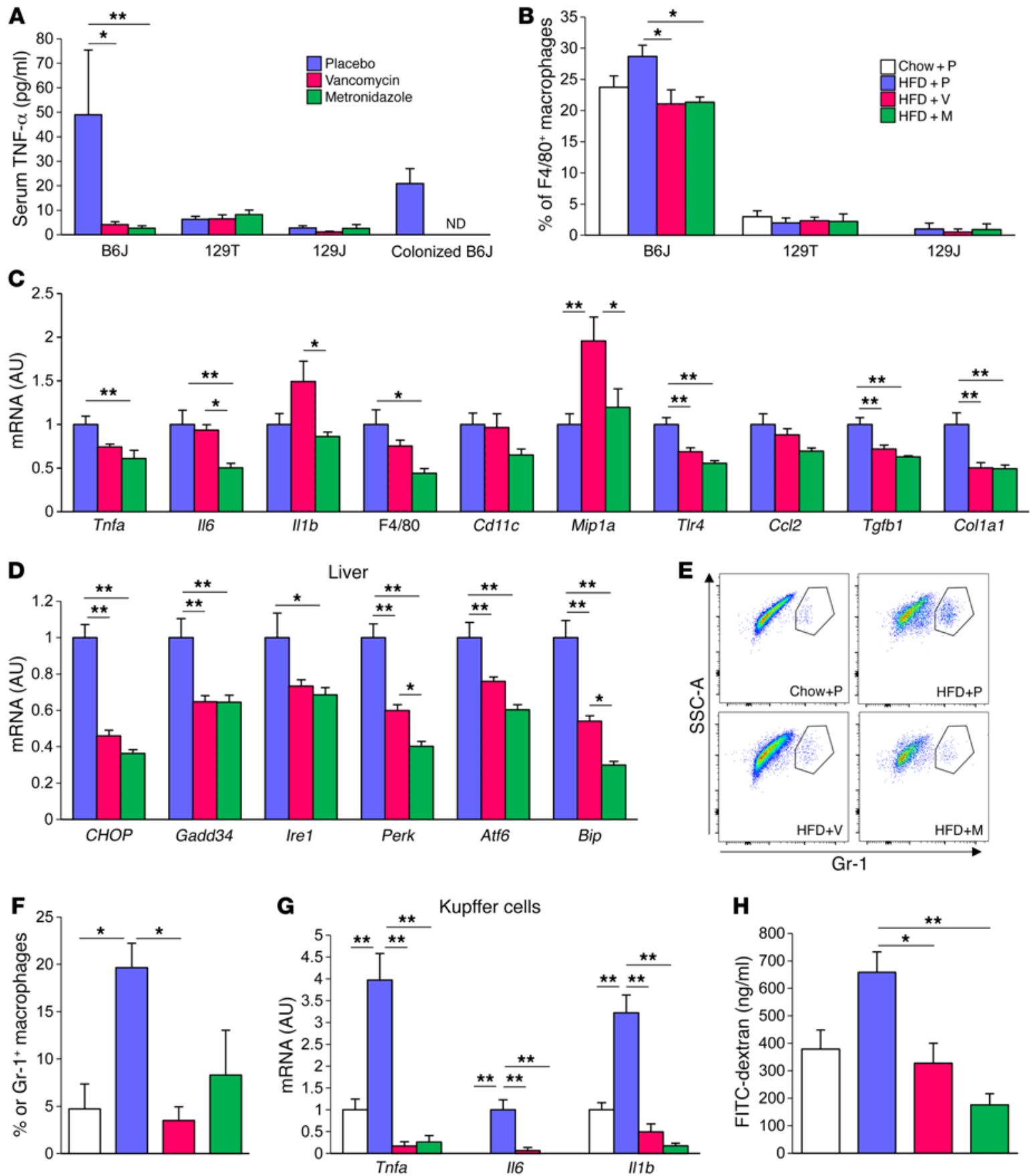

Figure 5. Gut microbiota modification by antibiotics ameliorates diet-induced inflammation in B6J mice. (A) Serum TNF- $\alpha$ levels of HFD-fed mice treated with placebo, vancomycin, or metronidazole for 9 weeks and of HFD-fed, GF mice colonized with bacteria from placebo-, vancomycin-, or metronidazole-treated $(n=8)$ mice. (B) Percentage of F4/80+ macrophages in CD11b+CD11 ${ }^{+}$cells in lamina propria $(n=5)$. (C and $\left.\mathbf{D}\right)$ qPCR analysis of inflammatory markers (C) and ER stress markers (D) in the liver ( $n=8 ; 10$ weeks of antibiotic treatment). (E) Representative flow cytometric results for $\mathrm{Gr}-1^{+}$cells in the liver of 8-week-old chow-fed mice and 26-week-old HFD-fed B6) mice $(n=3)$ ( 20 weeks on the HFD; 1 week of antibiotic treatment). SSC, side scatter. (F) Percentage of $\mathrm{Gr}-1^{+}$macrophages in CD11b+F4/80+ Kupffer cells in the liver. (C) qPCR analysis of sorted Kupffer cells $(n=3)$ and $(\mathbf{H})$ FITC levels in serum of B6) mice after gavage administration of FITC-dextran $(n=3-4)$. ${ }^{*} P<0.05$ and ${ }^{* *} P<0.01$, by ANOVA, followed by Tukey-Kramer post-hoc.

Antibiotic treatment ameliorates HFD-induced inflammation in B6J mice. Low-grade inflammation in visceral adipose tissue and liver is one component of the pathophysiology of insulin resistance in obesity. In HFD-fed B6J mice, treatment with vancomycin or metronidazole dramatically decreased their elevated serum TNF- $\alpha$ levels. This decrease in TNF- $\alpha$ levels was also observed in GF B6J mice colonized with cecal bacteria from antibiotic-treated mice. By contrast, in the insulin-sensitive 129 substrains, TNF- $\alpha$ levels were low even on an HFD, and antibiotic treatment had no effect (Figure 5A).

Flow cytometric analysis of cells isolated from the lamina propria of the colon showed high levels of $\mathrm{CD} 11 \mathrm{c}^{+} \mathrm{CD} 11 \mathrm{~b}^{+} \mathrm{F} 4 / 8 \mathrm{O}^{+}$macrophages in B6J mice compared with levels in both 129 substrains 
(Figure 5B). $\mathrm{CD} 11 \mathrm{c}^{+} \mathrm{CD} 11 \mathrm{~b}^{+} \mathrm{F} 4 / 80^{-}$dendritic cells in the mesenteric lymph node (MLN) were also higher in B6J mice, whereas the $\mathrm{CD}^{+}$cell population in MLNs was lower in B6J mice compared with that detected in both 129 strains (Supplemental Figure 4, G and $\mathrm{H}$ ). In $\mathrm{B} 6 \mathrm{~J}$ mice, the macrophage population in lamina propria was further elevated in response to an HFD, and this increase was markedly blunted by antibiotic treatment. Again, these effects were not observed in mice of the 129J or 129T strains, which had lower proportions of these cells. Thus, the $\mathrm{CD} 11 \mathrm{c}^{+} \mathrm{CD} 11 \mathrm{~b}^{+} \mathrm{F} 4 / 80^{+}$ macrophage population in lamina propria is influenced by both diet and antibiotics in mice prone to metabolic syndrome and may play an important role in regulating diet-induced inflammation, but these effects were not observed in mice that appeared to be genetically resistant to developing metabolic syndrome.

The decreased tissue inflammation in the antibiotic-treated mice was also indicated by decreased expression of $I l 6$ and $I l 1 b$ in mesenteric adipose tissue in response to metronidazole treatment (Supplemental Figure 5A), with a similar trend for inflammatory marker gene expression in epididymal adipose tissue (Supplemental Figure 5B). We also observed a decreased number of crown-like structures in epididymal adipose tissue in the antibiotic-treated, HFD-fed B6J mice (Supplemental Figure 5C), and qPCR assessment revealed reduced expression of inflammatory genes in the colons of mice from the antibiotic-treated groups (Supplemental Figure 5D).

Another site of reduction in inflammatory gene expression in HFD-fed mice treated with antibiotics was the liver, with decreases of $30 \%$ to $50 \%$ in Tnfa, Il6, Gpf480 (encoding F4/80), and Tlr4 expression levels (Figure 5C). In addition, markers of ER stress, including CHOP1O, Gadd34, Ire1, Perk, Atf6, and Bip, were significantly reduced in the livers of the antibiotic-treated, HFD-fed B6J mice (Figure 5D). The major population of immunoregulatory cells in the liver is Kupffer cells. Flow cytometric analysis revealed that the proportion of inflammation-activated Gr-1-positive Kupffer cells was greatly increased by an HFD, and this was also decreased by antibiotic treatment (Figures $5, \mathrm{E}$ and $\mathrm{F}$ ). In addition, FACS-isolated Kupffer cells showed significantly increased levels of Tnfa, Il6, and Illb expression in HFD-fed mice as assessed by qPCR, and these levels were dramatically decreased in both vancomycin- and metronidazole-treated groups (Figure 5G). The effects of antibiotics on lowering inflammatory gene expression was most rapid in the liver, where these effects could be observed as early as 4 weeks into the HFD period (Supplemental Figure 8, A and B), a point at which no significant differences were observed in adipose tissue (Supplemental Figure 8C). Again, in both 129T and $129 \mathrm{~J}$ mice, antibiotic treatment had no effect on reducing inflammatory gene expression and, in some cases, paradoxically upregulated the expression of inflammatory genes in fat, liver, and colon (Supplemental Figures 6 and 7). Thus, antibiotic modification of the gut microbiome improved insulin signaling and glucose metabolism only in obesity- and diabetes-prone B6J mice, which was correlated with an improvement in inflammation in multiple tissues, especially liver.

Antibiotics decrease plasma bile acid metabolites. To further define the mechanisms of improved insulin sensitivity and decreased inflammation, we assessed the effect of antibiotics on gut epithelial permeability, liver triglyceride (TG) content, and bile acid metabolism, all of which have been linked to insulin resistance or inflammation. Mice fed an HFD had higher serum levels of FITC after FITC-dextran gavage, and these were restored to normal levels by either vancomycin or metronidazole treatment, suggesting that an HFD increased gut permeability and that this was reversed by antibiotic treatment (Figure $5 \mathrm{H}$ ). In contrast, neither vancomycin nor metronidazole treatment affected the expression of genes encoding tight-junction proteins in the colon, plasma endotoxin levels, or expression of LPS-binding protein $(L b p)$ in the liver, nor were there any changes in liver TG content (Supplemental Figure 8, D-G).

However, an HFD and antibiotic treatment had major effects on the levels of plasma bile acid metabolites (Figure 6A). Plasma bile acid profiles in B6J mice revealed that an HFD increased the proportion of cholic acid (CA) and DCA and decreased chenodeoxycholic acid (CDCA). Both vancomycin and metronidazole, on the other hand, reduced the proportion of DCA to very low levels, and metronidazole also reduced CA to chow diet levels (Figure 6A). On a chow diet, $129 \mathrm{~T}$ mice had a higher proportion of CA than did B6J mice, while 129J mice had a higher proportion of muricholic acid (MCA). Vancomycin and metronidazole also increased the proportion of MCA in 129 T mice, whereas in 129J mice, vancomycin increased CA, while metronidazole decreased it (Figure 6A). Compared with 129T mice, 129J mice also showed higher levels of MCA, CA, CDCA, lithocholic acid (LCA), and hyodeoxycholic/ ursodeoxycholic acid (HDCA/UDCA) (Supplemental Figure 9).

The inflammatory bile acids TDCA and DCA $(21,22)$ are produced by $7-\alpha$-dehydroxylation of taurocholic acid (TCA) and CA by intestinal bacteria, primarily Clostridium XI and Clostridium XIVa, which express the bacterial enzyme baiE (23) Analysis of both cecal and plasma bile acids revealed that antibiotic treatment of mice of all 3 strains greatly decreased TDCA and DCA levels (Figures 6, B and C, and Supplemental Figure 9). This was probably secondary to decreases of more than $99 \%$ in these Clostridial clusters in both vancomycin- and metronidazole-treated mice (Figure 6D), resulting in a marked decrease in baiE gene levels detectable in the feces (Figure 6E and Supplemental Figure 10A). To determine whether these changes in TDCA might be linked to changes in the inflammatory response, B6J mice were fed either normal chow or chow supplemented with TDCA (0.4\% wt/wt), after which peritoneal macrophages were isolated and stimulated in vitro with bacterial LPS. Indeed, macrophages from mice fed the TDCA-supplemented diet showed 3- to 4-fold higher responses of Tnfa, Il6, and Il1b mRNA in response to LPS stimulation compared with what was observed in macrophages from control mice (Figure 7A). Taken together, these data indicate that antibiotic treatment decreases the levels of bile acid-modifying bacteria, including the major bacterial bile acid dehydroxylase, thereby reducing serum levels of the proinflammatory bile acids TDCA and DCA and resulting in a decreased inflammatory response in $\mathrm{B} 6 \mathrm{~J}$ mice.

The role of the bile acid receptor TGR5 in antibiotic effects on inflammatory response. Bile acids exert their effects through the nuclear receptor FXR and the G protein-coupled membrane receptor TGR5 (also known as GPBAR1 [G protein-coupled bile acid receptor 1]; M-BAR [membrane-type receptor for bile acids]; or GPR131 [G protein-coupled receptor 131]) (24). Several studies have demonstrated that TGR5 is highly expressed in immune cells 
A
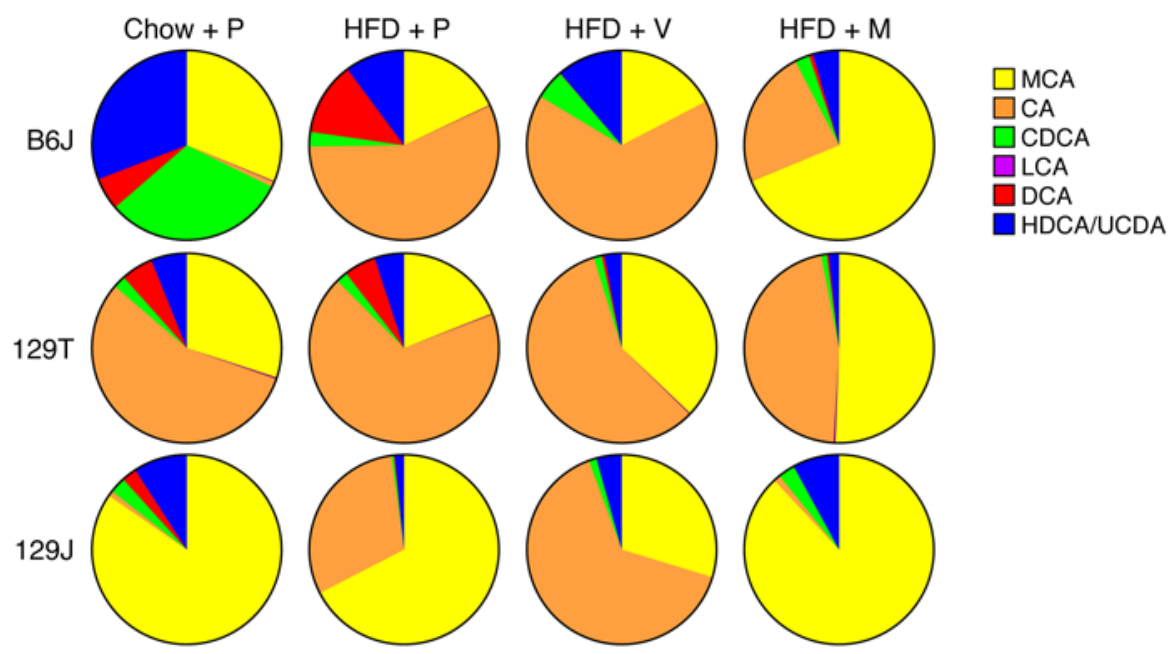

B
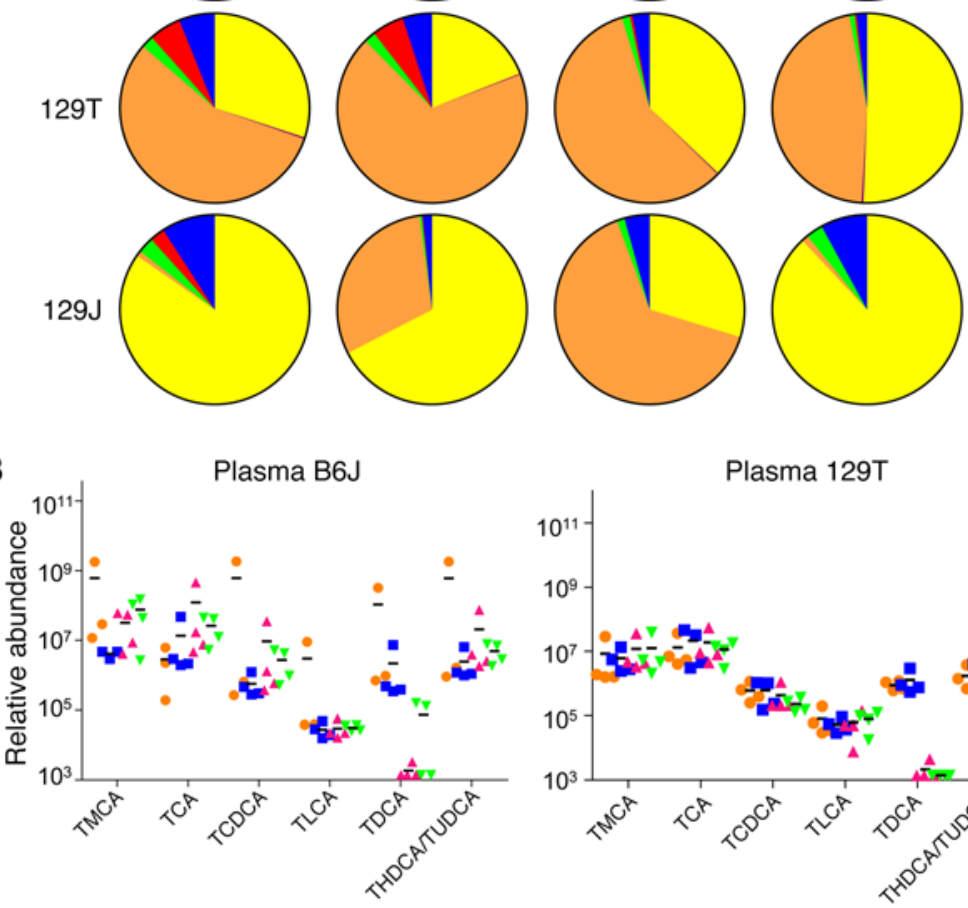

HDCA/UCDA
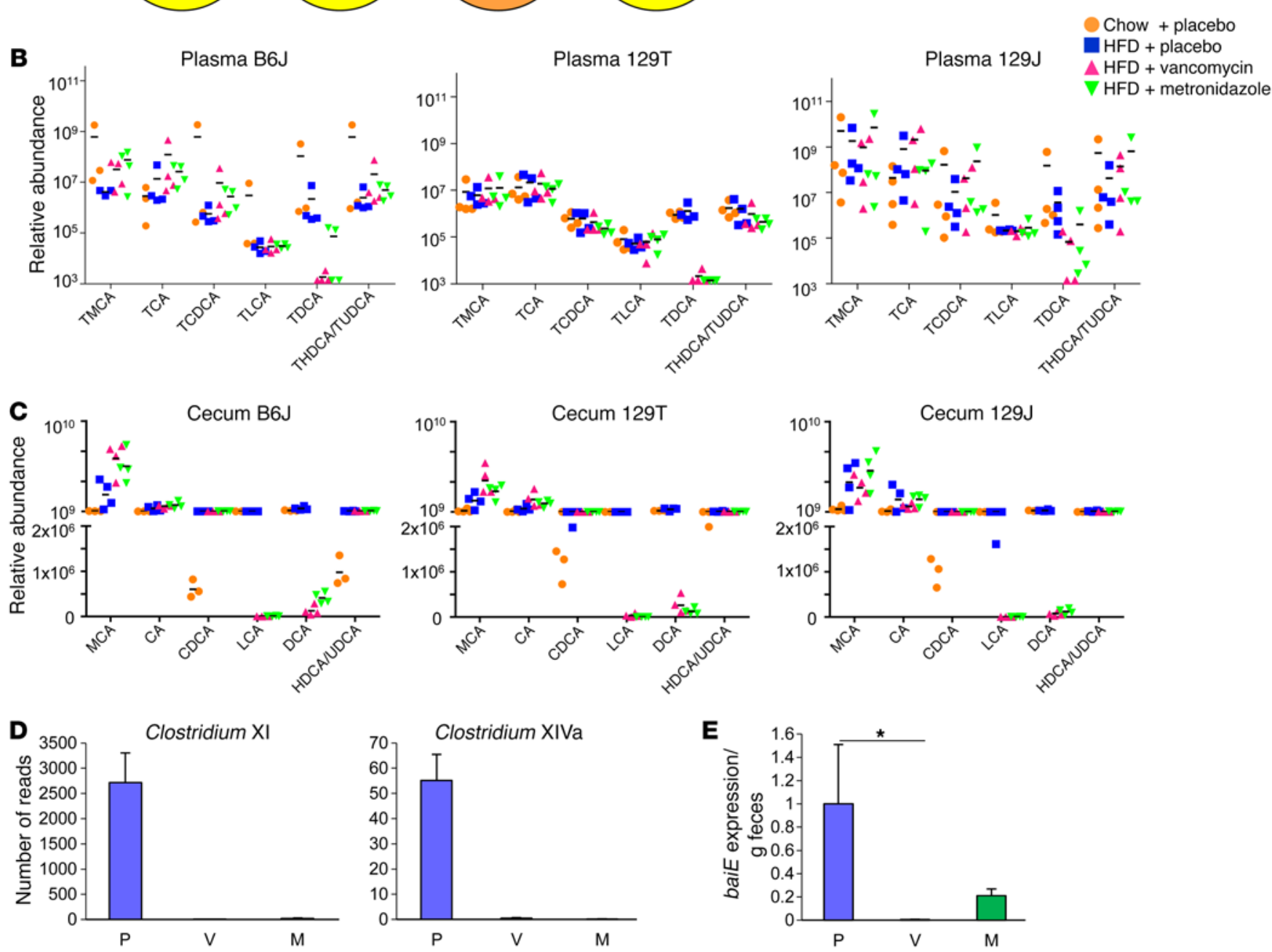

Figure 6. Antibiotics decrease plasma bile acid metabolites. (A) Total bile acid composition in the plasma of chow plus placebo-, HFD plus placebo-, HFD plus vancomycin-, and HFD plus metronidazole-treated B6), 129T, and 129J mice $(n=4)$ after 4 weeks of treatment. (B and C) Relative abundance of plasma tauro-conjugated bile acids (B) and cecum total bile acids (C) of individual mice from each treatment group. (D) Number of reads for Clostridium XI and Clostridium XIVa clusters $(n=8)$. (E) qPCR analysis for the baiE gene in feces collected from B6) mice treated for 8 weeks $(n=8)$. ${ }^{*} P<0.05$, by ANOVA, followed by Tukey-Kramer post-hoc.

and has an important role in regulating inflammation $(25,26)$. We hypothesized that the changes in bile acid composition by an HFD or antibiotic modification of gut microbiota may have an impact on TGR5 in the liver. Indeed, Western blot extracts revealed a decrease of more than $60 \%$ in TGR5 protein levels in the livers of mice of all 3 strains in response to an HFD (Figure 7B and Supplemental Figure 10B). Likewise, when Kupffer cells, which are the major cells in liver-expressing TGR5 (26), were isolated by cell 
A

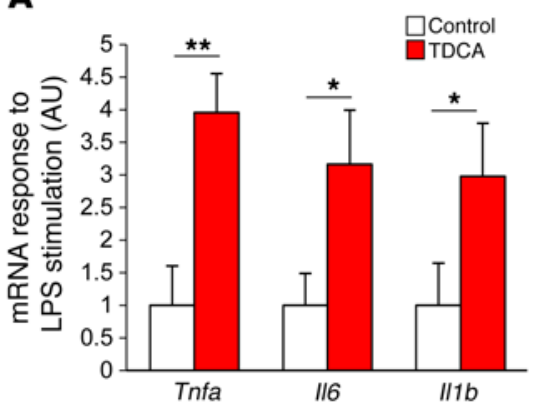

D
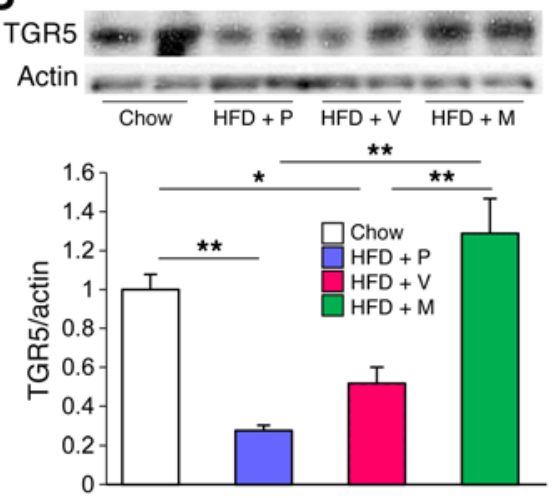

$\mathbf{F}$

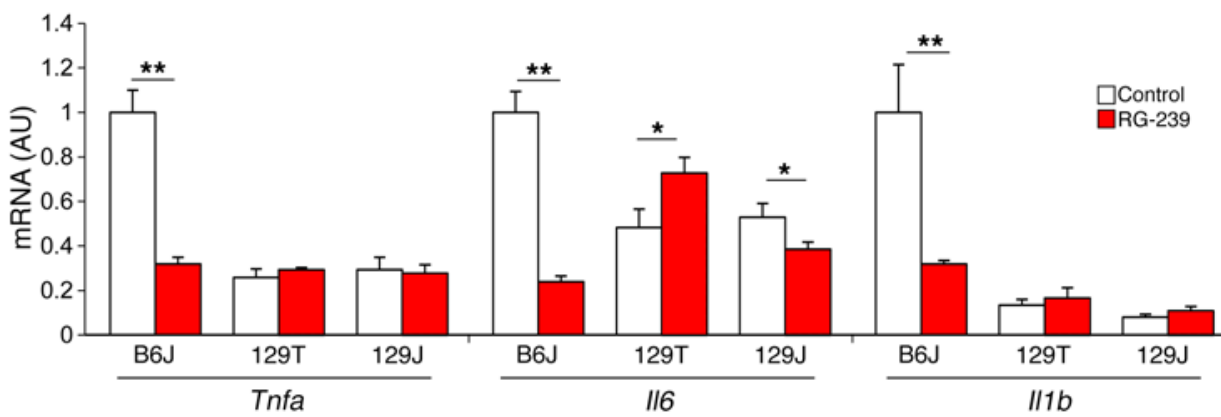

G

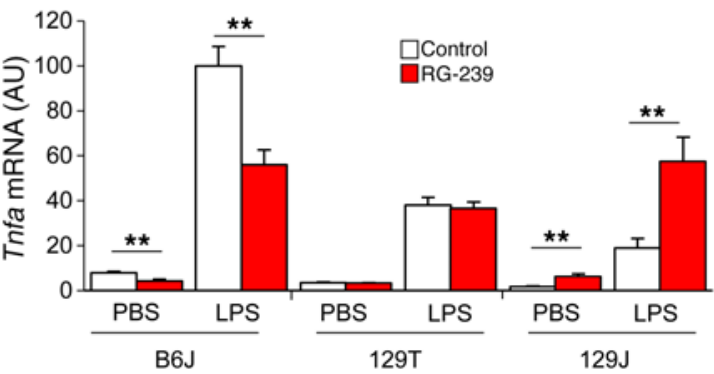

sorting, they showed a reduction of approximately $70 \%$ in $\operatorname{Tgr} 5$ mRNA in mice on an HFD (Figure 7C). In HFD-fed B6J mice, this decrease in TGR5 in the liver was partially restored by vancomycin and completely restored by metronidazole treatment (Figure 7D). Furthermore, when the gut microbiota of vancomycin- or metronidazole-treated mice were transferred to HFD-fed, GF B6J mice, a restoration of TGR5 levels was also observed (Figure 7E).

To understand the potential role of TGR5 activation in the metabolic responses, B6J, 129T, and 129J mice were treated with RG-239, a TGR5 receptor agonist (27). This resulted in a reduction in blood glucose levels in B6J mice, without any change in BW (Supplemental Figure 10, C and D). Mechanistically, this was associated with significant decreases in expression of the inflammatory genes Tnfa,

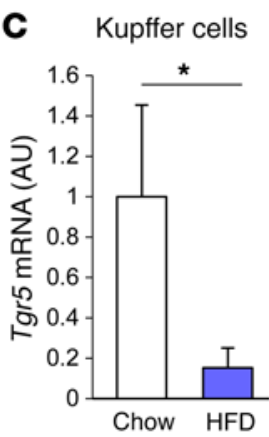

Figure 7. Modification of the gut microbiota by antibiotics restores bile acid receptor, TGR5 level in the liver. (A) qPCR for Tnfa, $/ 16$, and $/ 11 b$ mRNA in peritoneal macrophages collected from mice with or without TDCA treatment (0.4\%) for 3 weeks. Cells were stimulated with PBS or LPS $(10 \mathrm{ng} / \mathrm{ml})$ for 6 hours. Graph shows the fold change of relative expression levels after the stimulation $(n=5)$.

(B) Western blots for TCR5 in the liver of B6] mice on chow or an HFD for 22 weeks and quantitation of TCR5 protein normalized to actin ( $n=4$ per group). (C) qPCR for Tgr5 expression in Kupffer cells from mice fed chow or an HFD for 4 to 7 months $(n=8-9)$. (D) Western blots for TCR5 in the liver of 11-week-old B6] mice on chow or an HFD, with or without antibiotic treatment. Quantitation of TCR5 normalized to actin ( $n=4$ per group; 4 weeks on the HFD; 5 weeks on antibiotics). (E) Western blots for TCR5 in the liver of HFD-fed, GF B6J mice that received bacterial transfer from placebo-, vancomycin-, or metronidazole-treated mice and quantitation of TCR5 protein normalized to actin ( $n=5$-12 per group; 19 -week-old mice; 10 weeks on the HFD; 2 weeks after colonization). (F) qPCR for Tnfa, $/ 16$, and $111 \mathrm{~b}$ expression levels in the liver of mice treated with or without RG-239 (10 mg/ $\mathrm{kg} / \mathrm{d}$ ) for 2 weeks $(n=3-5)$. (G) qPCR for Tnfa expression of peritoneal macrophages collected from mice treated with or without RG-239 and stimulated in vitro with or without $10 \mathrm{ng} / \mathrm{ml}$ LPS for 6 hours $(n=4-6) .{ }^{*} P<0.05$ and ${ }^{*} P<0.01$, by unpaired, 2 -tailed $t$ test for (A-C and $\mathbf{E}-\mathbf{G})$ and by ANOVA, followed by Tukey-Kramer post-hoc for (D).
$I l 6$, and Il1b in the livers of B6J mice (Figure 7F). Peritoneal macrophages collected from TGR5 agonist-treated B6J mice also showed lower TNF- $\alpha$ expression in both the basal and LPS-stimulated states (Figure 7G). By contrast, no effect on inflammatory gene expression in liver was observed in response to the TGR5 agonist in either 129 strain (Figure 7F). Likewise, the TGR5 agonist had no effect on Tnfa expression in LPS-stimulated peritoneal macrophages in $129 \mathrm{~T}$ mice and tended to increase Tnfa expression in 129J mice (Figure 7G). Thus, an HFD decreased bile acid receptor TGR5 levels in the liver, which were restored by antibiotic modification of the gut microbiota. Antiinflammatory signaling through this increased level of TGR5 was specific to B6J mice, thus contributing to the decreased inflammation that was unique to mice of the B6J strain. 


\section{Discussion}

Multiple studies have established an important role of gut microbiota in the pathogenesis of a variety of diseases, including inflammatory bowel diseases, obesity, and type 2 diabetes. Exactly how gut microbiota modify disease risk in each of these cases involves important interactions with the host's genetic background $(8,14)$. We have recently shown that the development of metabolic phenotypes, such as weight gain, insulin resistance, and glucose intolerance, in response to an HFD is very different among inbred strains of mice and that this depends on a combination of factors including host genetics, response of gut microbiota to the dietary challenge, and other environmental factors including the site of rearing of the mice $(8,14)$. For example, B6J mice are prone to developing insulin resistance and metabolic syndrome when challenged with an HFD, whereas 129J mice from the same commercial breeder are resistant to insulin resistance and obesity under the same conditions (8). On the other hand, the mice of the genetically related $129 \mathrm{~T}$ substrain from another commercial breeder develop obesity and hepatosteatosis on an HFD but retain relative insulin sensitivity and have normal glucose tolerance (8). In the $129 \mathrm{~T}$ substrain, reprogramming of the gut microbiome by raising the mice in a new environment changed the response to an HFD, such that these mice became obesity resistant and had improved glucose tolerance, more closely resembling the $129 \mathrm{~J}$ mice. However, for $\mathrm{B} 6 \mathrm{~J}$ mice, changing the environment was not sufficient to change the response to an HFD, despite significant changes in the gut microbiome.

The aim of the present study was to further dissect the interactions between the host and the microbiome and begin to identify the mechanisms underlying the microbiome effects on insulin sensitivity and metabolic responses by analyzing how antibiotics, which cause dramatic remodeling of the microbiome, affect the response to an HFD in mice with differing genetic propensities for developing metabolic syndrome. For this purpose, we used 2 antibiotics that are also commonly used in patients with inflammatory bowel disease: vancomycin, a nonabsorbed antibiotic that targets gram-positive bacteria, and metronidazole, an absorbed broad-spectrum antibiotic that reduces anaerobic and facultative bacterial species.

In B6J mice, treatment with both antibiotics improved insulin signaling, insulin sensitivity, and glucose metabolism. This was associated with decreased systemic inflammation; decreased serum TNF- $\alpha$ levels; reduced markers of inflammation in adipose tissue, liver/Kupffer cells, and colon; and decreased macrophage infiltration into adipose tissue and the lamina propria of the colon. This appeared to be mediated, at least in part, through changes in bile acid metabolism and an upregulation of the bile acid receptor TGR5, which mediates antiinflammatory effects. The important role of host genetics in these responses is evidenced by the fact that these improvements were observed only in B6J mice and not in either 129 strain, despite similar effects of antibiotic treatment on the gut microbiome and bile acid metabolism.

We have previously shown that there are at least 3 factors that impact the different metabolic responses of B6J and 129 mice to an HFD. First, 129 mice have more brown/beige adipose tissue and more brown/beige adipocyte precursors than do B6J mice $(28,29)$. This results in an increased basal energy expenditure in 129 mice that protects against obesity. Second, expression of protein kinase $\mathrm{C} \delta(\mathrm{PKC} \delta$ ) in the liver is 2-fold higher in $\mathrm{B} 6$ mice than in 129 mice in the basal state and is further increased in B6J mice, but not 129 mice, in response to an HFD (30). PKC $\delta$, like other novel PKCs, can serine phosphorylate IRS-1 and the IR and promote insulin resistance. Third, and important in the context of this study, the inflammatory response to an HFD in adipose tissue is higher in B6J mice than in 129 mice $(8,31)$. Indeed, in the present study, we demonstrate that the insulin resistance-prone B6J mice have higher levels of inflammatory markers, not only in adipose tissue but also in the liver and in the intestinal lamina propria, when compared with both 129 strains. Antibiotic treatment greatly reduced inflammatory gene expression in B6J mice in all of these tissues, consistent with this being a significant factor in the changing response to an HFD and the changing microbiome. Indeed, the improvement in inflammation with antibiotic treatment was observed in the liver as early as 4 weeks after beginning an HFD, even at a point when no significant differences were observed in adipose tissue.

Multiple mechanisms appear to link gut microbiota to changes in metabolism and inflammation, including effects on gut development and permeability, metabolism of indigestible components of the diet, protection from colonization by pathogenic bacteria, and direct modulation of immune cell development and homeostasis (32). The most direct of these effects of gut microbiota are changes in gut permeability and the generation of endotoxins (33). In the present study, an HFD tended to increase gut permeability, which was reversed by antibiotic treatment, suggesting that gut permeability may play a role in the antibiotic response. However, antibiotics improved insulin sensitivity in HFD-fed B6J mice, without producing any differences in expression of the genes for tight-junction-related proteins, changes in plasma endotoxin levels, or changes in expression levels of LBP in the liver, suggesting that other mechanisms may be more important in the observed decrease in systemic inflammation.

Recent studies have demonstrated that the gut microbiota can affect the metabolism of a large number of substances, especially bile acids, which can secondarily affect the immune system and the physiological response to an HFD (34). As part of normal physiology, hepatocytes produce CA and CDCA from cholesterol. These lipid-soluble bile acids are conjugated to glycine or taurine molecules to form water-soluble primary bile acids, which are stored in the gall bladder and released during digestion into the duodenum. In the lower small intestine and colon, bacteria dehydroxylate the primary bile salts to form secondary bile salts, which are actively reabsorbed along the proximal and distal ileum into the hepatic portal circulation. Bacteria can also deconjugate some of the primary and secondary conjugated bile salts back to lipid-soluble bile acids, which are passively absorbed into the hepatic portal circulation. In all, $95 \%$ of the bile acids that are delivered to the duodenum are recycled by this enterohepatic circulation.

In the present study, we found that an HFD significantly increased the proportions of both CA and DCA and decreased the levels of CDCA in the circulation of the host animals, while antibiotic treatment reduced both DCA and TDCA levels. DCA and TDCA are produced in the intestine from CA and TCA primarily by 2 bacterial clusters, Clostridium XI and Clostridium XIVa (23). Treatment with either vancomycin or metronidazole causes 
a decrease of more than $99 \%$ in the levels of bacteria in these clusters. This is accompanied by a parallel decrease in expression levels of the bacterial gene baiE, which encodes the gene responsible for $7 \alpha$-dehydroxylation, which is required for the production of these bile acids. Parallel decreases in DCA and TDCA in the intestinal lumen occur as well. Although, in all 3 strains of mice, antibiotics reduced the numbers of these bacteria and the levels of baiE gene expression and vancomycin and metronidazole decreased their TDCA levels, it is important to note that the serum bile acid composition is very different in these 3 strains of mice, as B6J mice have lower plasma levels of total CA, MCA and CDCA compared with 129J mice, while 129J mice have higher levels of total lithocholic acid (LCA).

Previous studies have reported that DCA can promote intestinal inflammation by inducing reactive oxygen and nitrogen species and activating NF- $\kappa \mathrm{B}$ signaling in intestinal epithelial cells (22). This bile acid has an important effect on the inflammatory response in $\mathrm{B} 6 \mathrm{~J}$ mice as demonstrated by the finding that macrophages from the mice fed a TDCA-supplemented diet show a greater response of TNF- $\alpha$ to LPS treatment. An increase in DCA in response to an HFD has also been suggested to play a role in the development of liver cancer (35). We found that, while all 3 strains of mice had between $2.6 \%$ and $5.5 \%$ of plasma bile acids in the form of DCA conjugates on a chow diet, only B6J mice showed an increase in the proinflammatory bile acid DCA on an HFD, and this increase was completely blocked by antibiotic treatment. Thus, at least one component of the antibiotic effect to decrease inflammation in B6J mice is that of decreasing the bile acid modifying bacteria responsible for the production of DCA and its conjugates. Both vancomycin and metronidazole are currently used for $C$. difficile-associated diseases such as pseudomembranous colitis. This reduction in DCA and TDCA might, at least a part, be another mechanism of these treatments.

There are 2 major receptors of bile acids: the nuclear receptor FXR and the cell membrane receptor TGR5 (36). Whereas FXR signaling is known to regulate bile acid (37) and lipid metabolism (38), the role of TGR5 is less clear. TGR5 activation has been shown to induce energy expenditure in brown adipose tissue (39) and glucagon-like peptide 1 (GLP-1) secretion in the intestine (40). TGR5 is also highly expressed on immune cells $(25,26)$ and has been suggested to have antiinflammatory effects (41). Indeed, recent studies have shown that obese mice lacking TGR5 in macrophages exhibit enhanced adipose inflammation and enhanced insulin resistance compared with control obese animals, while pharmacological activation of TGR5 can decrease LPS-induced chemokine expression in macrophages (42).

In the present study, the change of inflammation in B6J mice appeared to be, at least in part, the response to bile acids acting through the TGR5 receptor; however, this response was also partly dependent on the host's genetic background. Thus, there was a marked reduction in TGR5 levels in the liver in all 3 strains of mice when challenged with an HFD. This was reversed by antibiotic treatment, especially metronidazole, and was coincident with a significant reduction in intraintestinal and circulating levels of TDCA and DCA. Since secondary bile acids like DCA have a high affinity for TGR5 (43), the effect of antibiotics to decrease DCA and TDCA levels may contribute to the restoration of TGR5 protein levels in the liver. Consistent with previous studies (26), we found that in liver, TGR5 receptors were expressed primarily on Kupffer cells. We also found that the TGR5-selective agonist RG-239 could produce an antiinflammatory effect. Interestingly, however, although hepatic TGR5 levels were similar in all 3 mouse strains, the antiinflammatory effect of RG-239 was observed only in B6J mice, again indicating the important role of the host's genetic background and the inflammatory potential in the interactions between the gut microbiome and metabolism. In future studies, it would be of interest to determine the effects of inactivation of the Tgr5 gene in each of these mouse strains.

At a molecular level, one of the most striking findings of this study was the improvement in insulin signaling in liver, fat, and muscle of the antibiotic-treated animals. Vancomycin enhanced the phosphorylation of AKT, ERK, and the IR in B6J mice, even in the basal state, while metronidazole increased AKT phosphorylation only in response to insulin. Some of these changes in signaling were reproduced by fecal transplantation, suggesting that they were due to changes in gut microbiota. While it is possible that the enhanced insulin response was due to a lower level of systemic inflammation created by the beneficial microbial community, the mechanism underlying the increase in basal insulin signaling remains unknown, as this was not observed in the microbiota transfer experiment. It is possible that the enhanced basal signaling was due to some direct metabolic product of the altered microbial community or that it required a host-microbiome interaction that was not reproduced in the microbiome transfer. Alterations of the gut microbiome by vancomycin have been reported to enhance GLP-1 secretion in B6J mice (6), however, we did not observe any significant difference in serum GLP-1 levels (data not shown) or any difference in serum insulin levels in the mice treated with antibiotics, suggesting that the altered insulin signaling was not simply due to increased basal insulin levels.

In addition to our study showing a reduction of inflammatory bacterial metabolites with antibiotic treatment, others have shown that, under some circumstances, antibiotic treatment can also decrease HFD-induced endotoxemia in obese mice, which may have beneficial effects on glucose metabolism (44). However, long-term antibiotic treatment would probably not be an acceptable approach to treating obesity-associated insulin resistance and metabolic syndrome (45). In fact, low-dose antibiotic treatment in humans in early life may actually increase the risk of metabolic dysfunction in adulthood (46). Likewise, short-term treatment of obese humans with metabolic syndrome with vancomycin appears to decrease, rather than increase, systemic insulin sensitivity (47). This is consistent with our findings that the interaction between the gut microbiome and insulin sensitivity is very complex and depends on multiple host factors as well as the environment.

In summary, antibiotic treatment has profound effects on the gut microbiome that result in changes in bile acid metabolism, inflammatory and insulin signaling, and glucose homeostasis. The exact composition of the resulting bacterial community and bile acid metabolites as well as the downstream responses vary greatly, however, depending on host genetics and inflammatory potential. In inflammation-prone hosts, antibiotics can reduce inflammatory metabolites, such as DCA and TDCA, and increase antiinflammatory TGR5 signaling. This is associated with improved insulin sig- 
naling and improved glucose metabolism in these animals. However, the effects of bacterial modification depend on the mouse strain and the host's genetic inflammatory potential. Understanding the important interactions and the molecular pathways that link the gut microbiome and host genetics is critical to dissecting the role of the microbiome in determining the metabolic response.

\section{Methods}

Additional details are provided in the Supplemental Methods.

Mouse Procedures. B6J and 129J mice were purchased from The Jackson Laboratory, and 129T mice were purchased from Taconic Farms. Mice were maintained on either a normal chow diet containing $22 \%$ of calories from fat, $23 \%$ from protein, and 55\% from carbohydrates (Mouse Diet 9F 5020; PharmaServ) or an HFD (Open Source Diet, D12492; Research Diets) containing 60\% of calories from fat, $20 \%$ from protein, and $20 \%$ from carbohydrates. For antibiotic treatment, 6-week-old mice were treated with either placebo, vancomycin $(1 \mathrm{~g} / \mathrm{l})$, or metronidazole $(1 \mathrm{~g} / \mathrm{l})$ (Sigma-Aldrich) in the drinking water, then started on an HFD from age 7 to 16 weeks (Figure 2A). For bacterial transfer experiments, recipient mice were pretreated with a mixture of ampicillin $(1 \mathrm{~g} / \mathrm{l})$, vancomycin $(0.5 \mathrm{~g} / \mathrm{l})$, neomycin $(1 \mathrm{~g} / \mathrm{l})$, and metronidazole $(1 \mathrm{~g} / \mathrm{l})$ via the drinking water for 3 days prior to the transfer. Cecal contents were collected from donor mice immediately after euthanasia, suspended with PBS, and filtered through a $40-\mu \mathrm{m}$ cell strainer. Bacterial transfer was performed by gastric gavage with $200 \mu$ l of diluted cecal contents. RG-239 was purchased from SigmaAldrich and used to supplement the chow diet $(10 \mathrm{mg} / \mathrm{kg} / \mathrm{d})$.

GF B6J mice were obtained from the gnotobiotic core facility of Brigham and Women's Hospital. Bacterial transfer was performed as described in the Supplemental Methods.

Flow cytometric analysis. For flow cytometry, cells were isolated from lamina propria as described in the Supplemental Methods and analyzed by flow cytometry using a BD LSR II Flow Cytometer (BD Biosciences). Flow cytometry for Kupffer cell sorting was performed as previously described (48) using a FACSAria cell sorter (BD Biosciences).

Bile acids analysis. Bile acids were analyzed using a Nexera X2 U-HPLC system (Shimadzu Scientific Instruments) coupled to a $\mathrm{Q}$ Exactive Orbitrap Mass Spectrometer (Thermo Fisher Scientific). Mass spectrometric analyses were carried out using electrospray ionization in the negative ion mode with full-scan analysis over $\mathrm{m} / \mathrm{z}$ 200-550 at 70,000 resolution and a $3-\mathrm{Hz}$ data acquisition rate. Targeted processing was conducted using TraceFinder software, version 3.1 (Thermo Fisher Scientific).

$16 S$ rRNA sequencing analysis. DNA was extracted from mouse feces using a MO BIO Fecal DNA Extraction Kit (MO BIO Laboratories Inc.). A multiplexed amplicon library covering the $16 \mathrm{~S}$ rDNA gene V4 region was generated from DNA-extracted samples. Reads were generated on a MiSeq instrument from the amplicon library and clus- tered into operational taxonomic units (OTUs). A total of 5,606,228 sequence reads were generated, corresponding to an average of 72,109 (range, 30,087-161,983) reads per sample. Differences in microbial community structure were visualized using phylogenetic methods. The number of OTUs per sample was then scaled so that each sample had the same mean, filtered to only include OTUs that were present at $0.1 \%$ of the total counts in at least 6 samples, log transformed using $\log _{2}$ (count +0.5 ), and plotted in PCA space using R software. $16 \mathrm{~S}$ rRNA data sets were deposited in the NCBI's Sequence Read Archive (SRA) database (accession no. SRP080944).

Statistics. Statistical significance was evaluated using ANOVA, followed by a Tukey-Kramer post-hoc, Bonferroni's post-hoc, or unpaired, 2-tailed $t$ test where appropriate. A $P$ value of less than 0.05 was considered significant. Results are presented as the mean \pm SEM.

Study approval. All experiments complied with regulations and ethics guidelines of the National Institute of Health and were approved by the IACUC of the Joslin Diabetes Center (no. 97-05) and Harvard Medical School (no. 05131).

\section{Author contributions}

SF designed and performed experiments and wrote the manuscript. SU, M Sakaguchi, and SS contributed to discussions. CC performed bile acids analysis. SD, MK, EA, and M Soto performed experiments. JMD performed data analysis. NL, GG, and LB conducted the microbiome analyses. CRK designed experiments and wrote the manuscript. CRK is the guarantor of this work and, as such, had full access to all the data and takes responsibility for the integrity of the data and the accuracy of the data analysis.

\section{Acknowledgments}

We thank Christie Penniman, Vladimir Yeliseyev, Luxue Deng, and Hui Pan for their technical assistance. This work was supported by grants from the NIH (R01DK031036 and R01DK033201, to CRK); a Mary K. Iacocca Professorship (to CRK); and K-12HD000850 from NICHD (to SS). SF was supported by a Sunstar Foundation Postdoctoral Fellowship. SU was supported by iMed, the Helmholtz Initiative on Personalized Medicine, and the German Center for Diabetes Research (DZD). The authors also thank the Joslin DRC Bioinformatics Core (NIH grant P30DK036836); as the Joslin Animal Physiology Core; the Joslin Flow Cytometry Core; the Joslin Specialized Assay Core; and the Brigham and Women's Hospital, Harvard Digestive Diseases Center for Clinical and Translational Metagenomics (NIH grant P3ODK034854) for their core support.

Address correspondence to: C. Ronald Kahn, Joslin Diabetes Center, One Joslin Place, Boston, Massachusetts 02215, USA. Phone: 617.309.2635; E-mail: C.Ronald.Kahn@joslin.harvard.edu.
1. Velagapudi VR, et al. The gut microbiota modulates host energy and lipid metabolism in mice. J Lipid Res. 2010;51(5):1101-1112.

2. Le Chatelier E, et al. Richness of human gut microbiome correlates with metabolic markers. Nature. 2013;500(7464):541-546.

3. Turnbaugh PJ, Ley RE, Mahowald MA, Magrini V, Mardis ER, Gordon JI. An obesity-associated gut microbiome with increased capacity for energy harvest. Nature. 2006;444(7122):1027-1031.

4. Ridaura VK, et al. Gut microbiota from twins discordant for obesity modulate metabolism in mice. Science. 2013;341(6150):1241214.

5. Cho I, et al. Antibiotics in early life alter the murine colonic microbiome and adiposity. Nature. 2012;488(7413):621-626.
6. Hwang I, et al. Alteration of gut microbiota by vancomycin and bacitracin improves insulin resistance via glucagon-like peptide 1 in dietinduced obesity. FASEB J. 2015;29(6):2397-2411.

7. Henao-Mejia J, et al. Inflammasome-mediated dysbiosis regulates progression of NAFLD and obesity. Nature. 2012;482(7384):179-185.

8. Ussar S, et al. Interactions between gut microbi- 
ota, host genetics and diet modulate the predisposition to obesity and metabolic syndrome. Cell Metab. 2015;22(3):516-530.

9. Carmody RN, et al. Diet dominates host genotype in shaping the murine gut microbiota. Cell Host Microbe. 2015;17(1):72-84.

10. Cani PD, et al. Changes in gut microbiota control metabolic endotoxemia-induced inflammation in high-fat diet-induced obesity and diabetes in mice. Diabetes. 2008;57(6):1470-1481.

11. Canfora EE, Jocken JW, Blaak EE. Short-chain fatty acids in control of body weight and insulin sensitivity. Nat Rev Endocrinol. 2015;11(10):577-591.

12. Joyce $\mathrm{SA}$, et al. Regulation of host weight gain and lipid metabolism by bacterial bile acid modification in the gut. Proc Natl Acad Sci U S A. 2014;111(20):7421-7426.

13. Rescigno M. Intestinal microbiota and its effects on the immune system. Cell Microbiol. 2014;16(7):1004-1013.

14. Parks BW, et al. Genetic control of obesity and gut microbiota composition in response to high-fat, high-sucrose diet in mice. Cell Metab. 2013;17(1):141-152.

15. Bouchard C, et al. The response to long-term overfeeding in identical twins. N Engl JMed. 1990;322(21):1477-1482.

16. Hotamisligil GS. Inflammation and metabolic disorders. Nature. 2006;444(7121):860-867.

17. Osborn O, Olefsky JM. The cellular and signaling networks linking the immune system and metabolism in disease. Nat Med. 2012;18(3):363-374.

18. Goldfine AB, et al. Use of salsalate to target inflammation in the treatment of insulin resistance and type 2 diabetes. Clin Transl Sci. 2008;1(1):36-43.

19. Cotillard A, et al. Dietary intervention impact on gut microbial gene richness. Nature. 2013;500(7464):585-588.

20. Huang EY, Leone VA, Devkota S, Wang Y, Brady MJ, Chang EB. Composition of dietary fat source shapes gut microbiota architecture and alters host inflammatory mediators in mouse adipose tissue. JPEN J Parenter Enteral Nutr. 2013;37(6):746-754.

21. Mühlbauer M, et al. Differential effects of deoxycholic acid and taurodeoxycholic acid on NF-kappa B signal transduction and IL-8 gene expression in colonic epithelial cells. Am JPhysiol Gastrointest Liver Physiol. 2004;286(6):G1000-G1008.

22. Payne CM, et al. Deoxycholate induces mitochondrial oxidative stress and activates NF-kappaB through multiple mechanisms in HCT-116 colon epithelial cells. Carcinogenesis. 2007;28(1):215-222.

23. Ridlon JM, Kang DJ, Hylemon PB. Bile salt biotransformations by human intestinal bacteria. JLipid Res. 2006;47(2):241-259.

24. Thomas C, Pellicciari R, Pruzanski M, Auwerx J, Schoonjans K. Targeting bile-acid signalling for metabolic diseases. Nat Rev Drug Discov. 2008;7(8):678-693.

25. Pols TW, et al. TGR5 activation inhibits atherosclerosis by reducing macrophage inflammation and lipid loading. Cell Metab. 2011;14(6):747-757.

26. Keitel V, Donner M, Winandy S, Kubitz R, Häussinger D. Expression and function of the bile acid receptor TGR5 in Kupffer cells. Biochem Biophys Res Commun. 2008;372(1):78-84.

27. Genet C, et al. Structure-activity relationship study of betulinic acid, a novel and selective TGR5 agonist, and its synthetic derivatives: potential impact in diabetes. JMed Chem. 2010;53(1):178-190.

28. Almind K, Manieri M, Sivitz WI, Cinti S, Kahn CR. Ectopic brown adipose tissue in muscle provides a mechanism for differences in risk of metabolic syndrome in mice. Proc Natl Acad Sci U S A. 2007;104(7):2366-2371.

29. Schulz TJ, et al. Identification of inducible brown adipocyte progenitors residing in skeletal muscle and white fat. Proc Natl Acad Sci U S A. 2011;108(1):143-148.

30. Bezy O, et al. PKC $\delta$ regulates hepatic insulin sensitivity and hepatosteatosis in mice and humans. J Clin Invest. 2011;121(6):2504-2517.

31. Mori MA, et al. A systems biology approach identifies inflammatory abnormalities between mouse strains prior to development of metabolic disease. Diabetes. 2010;59(11):2960-2971.

32. Sommer F, Bäckhed F. The gut microbiotamasters of host development and physiology. Nat Rev Microbiol. 2013;11(4):227-238.

33. Luche E, et al. Metabolic endotoxemia directly increases the proliferation of adipocyte precursors at the onset of metabolic diseases through a CD14-dependent mechanism. Mol Metab. 2013;2(3):281-291.

34. Wikoff WR, et al. Metabolomics analysis reveals large effects of gut microflora on mammalian blood metabolites. Proc Natl Acad Sci U S A. 2009;106(10):3698-3703.
35. Yoshimoto S, et al. Obesity-induced gut microbial metabolite promotes liver cancer through senescence secretome. Nature. 2013;499(7456):97-101.

36. Kuipers F, Bloks VW, Groen AK. Beyond intestinal soap--bile acids in metabolic control. Nat Rev Endocrinol. 2014;10(8):488-498.

37. Lu TT, et al. Molecular basis for feedback regulation of bile acid synthesis by nuclear receptors. Mol Cell. 2000;6(3):507-515.

38. Jiang C, et al. Intestinal farnesoid $\mathrm{X}$ receptor signaling promotes nonalcoholic fatty liver disease. JClin Invest. 2015;125(1):386-402.

39. Watanabe $\mathrm{M}$, et al. Bile acids induce energy expenditure by promoting intracellular thyroid hormone activation. Nature. 2006;439(7075):484-489.

40. Thomas C, et al. TGR5-mediated bile acid sensing controls glucose homeostasis. Cell Metab. 2009;10(3):167-177.

41. Perino A, Schoonjans K. TGR5 and immunometabolism: Insights from physiology and pharmacology. Trends Pharmacol Sci. 2015;36(12):847-857.

42. Perino A, Pols TW, Nomura M, Stein S, Pellicciari $\mathrm{R}$, Schoonjans K. TGR5 reduces macrophage migration through mTOR-induced $\mathrm{C} / \mathrm{EBP} \beta$ differential translation. J Clin Invest. 2014;124(12):5424-5436.

43. Kawamata Y, et al. A G protein-coupled receptor responsive to bile acids. J Biol Chem. 2003;278(11):9435-9440.

44. Carvalho BM, et al. Modulation of gut microbiota by antibiotics improves insulin signalling in high-fat fed mice. Diabetologia. 2012;55(10):2823-2834.

45. Keeney KM, Yurist-Doutsch S, Arrieta MC, Finlay BB. Effects of antibiotics on human microbiota and subsequent disease. Annu Rev Microbiol. 2014;68:217-235.

46. Cox LM, et al. Altering the intestinal microbiota during a critical developmental window has lasting metabolic consequences. Cell. 2014;158(4):705-721.

47. Vrieze A, et al. Impact of oral vancomycin on gut microbiota, bile acid metabolism, and insulin sensitivity. J Hepatol. 2014;60(4):824-831.

48. Kohli R, et al. High-fructose, medium chain trans fat diet induces liver fibrosis and elevates plasma coenzyme $\mathrm{Q} 9$ in a novel murine model of obesity and nonalcoholic steatohepatitis. Hepatology. 2010;52(3):934-944. 